\title{
DSIMA: A testbed for the quantitative analysis of interaction models within distribution networks
}

\author{
S. Mathieu, Q. Louveaux, D. Ernst, B. Cornélusse* \\ Department of Electrical Engineering and Computer Science, \\ University of Liège, Liège 4000, Belgium
}

\begin{abstract}
This article proposes an open-source testbed to simulate interaction models governing the exchange of flexibility services located within a distribution network. The testbed is an agent-based system in which the distribution system operator, the transmission system operator, producers and retailers take their decisions based on mixed-integer linear programs. This testbed helps to highlight the characteristics of an interaction model, its benefits for the agents and ease the detection of unwanted or abusive behaviors which decreases the welfare. The testbed is implemented in Python and the optimization problems are encoded in the modeling language ZIMPL. A web interface is coupled with an instance generator using macroscopic parameters of the system such as the total power production. This tested is therefore well suited to test the implemented interaction models on various scenarios and extend the implementation to other models. Five interaction models devised with industrial partners are simulated over a year on a 75-bus test system. Simulations show that interaction models relying on active network management, in the way they have been stated, lead to large welfare even though they suffer from a lack of coordination between the DSO and the TSO. A conservative interaction model restricting grid users to an access range computed ahead of time to prevent any congestion avoids shedding distributed generation but restrains considerably the amount of distributed production.
\end{abstract}

Keywords: Market design, load flexibility, demand-side management, agent-based model

\footnotetext{
* Corresponding author.

Email address: sebastien.mathieu@ulg.ac.be, bertrand.cornelusse@ulg.ac.be, dernst@ulg.ac.be, q.louveaux@ulg.ac.be (S. Mathieu, Q. Louveaux, D. Ernst, B. Cornélusse)
}

Preprint submitted to Sustainable Energy, Grids and Networks 


\section{Introduction}

In the previous decades, the operation of the distribution network did not rely on flexibility of the production or consumption. With the ongoing trend for renewable energies, the needs of investments to increase distribution network capacity could become too expensive to be satisfied. Distribution System Operators (DSO) need to access to flexibility services in order to reduce the investment needs. The literature contains many papers showing the benefits of using such flexibility in distribution networks $[1,2,3,4]$ and the methods to deliver this flexibility $[5,6,7,8]$. However, few studies have been performed on the way the flexibility in distribution network can be exchanged as a commodity in an unbundled electric system. As the DSO does not own the assets able to provide flexibility, it has to procure flexibility by contracting it to a Flexibility Services Provider (FSP) which may be a producer, a retailer, an aggregator, etc. One current challenge faced by regulators is to design the legislative framework or interaction model in which actors exchange flexibility services located within a distribution network.

This paper proposes an open-source testbed to simulate candidate interaction models and perform this kind of comparison. In this testbed, the distribution network is modeled up to its interface to the high voltage network and down to medium voltage-low voltage transformers, i.e. low voltage sub-networks are aggregated. We work in a short term context, from a few days ahead until settlement. We thus do not consider structural changes of the system. The simulation is performed by an agent-based method considering as agents the distribution system operator, the transmission system operator, producers and retailers with the consumers they serve. Every agent solves an optimization problem at every decision stage in order to maximize its individual objective. The system operators desire to ensure the safety of their system at the minimum costs while producers and retailers maximize their profit by selling or retailing electricity and providing flexibility services. The major contribution of this paper is the complete formalization and simulation of the interactions between the stakeholders of a distribution system exchanging flexibility. These exchanges are described by five interaction models devised with industrial partners and their impact is evaluated through a measure of the social welfare, the repartition of the welfare between the actors, and a measure of the service level that is reached. The behavior of each agent under a specific interaction model can be observed and weaknesses or abuses of an interaction model can be highlighted.

The complex interactions that happen in electricity markets demand a flexible computational environment where designs can be tested and sensitivities to power system and market rule changes can be explored [9]. Agent-based modeling has been extensively used in the literature to model electric systems $[10,11,12,13]$. The OPTIMATE open platform is an example of an agentbased tool used in power systems. It can be used for evaluating and comparing various existing and potential new market designs in several regional European power markets [14]. Another example uses an agent based approach for evaluating the effects of the integration of flexible loads in the secondary reserve 
market [15]. Alternative techniques for carrying quantitative analysis in power systems are based on the computation of market equilibria. For instance the PRIMES model computes a market equilibrium solution in the European Union member states taking into consideration market economics, industry structure, energy/environmental policies and regulation [16]. Note that the references here above are related to the wholesale energy market, whereas the present paper applies agent-based modeling to interaction models within distribution systems.

Several European projects study the interactions models to integrate flexibility services in the present electric system. The ADDRESS project aims at developing a comprehensive commercial and technical framework for the development of active demand in the smart grids of the future [17]. This project focuses on questions such as which information should be available for each actor or which reference consumption to choose. Other projects in Europe rely on the deployment of demand side management such as GREDOR [18], iPower [19], EvolvDSO [20], LINEAR [21], ADINE [22], Local Load Management [23], Nice Grid [24], Optimate [25]. The testbed introduced in this paper is a convenient tool to test the ideas proposed in the previous projects.

The name of the testbed, DSIMA, stands for Distribution System Interaction Model Analysis. This testbed is a framework is composed of an instance generator, a simulator and a web-based user interface. These three modules allow various compromises between versatility and ease of modification. For instance, the behavior of an agent can easily be changed by changing its optimization problems written in the user-friendly modeling language ZIMPL, see Figure 1 for an example. More flexibility can be obtained by modifying the open-source Python 3 code available at the address http://www.montefiore.ulg.ac.be/ dsima/. In this testbed, the network is modeled as a network flow model. This model considers only active power flows and therefore cannot grasp voltage issues. The extension to an optimal power flow model over multiple time periods leads to non-linear and non convex problems. These problems are computationally challenging, see [26] for a review of the current techniques to solve this kind of dynamic optimal power flows. Integrating OPF would make the testbed more complex to use, increase the computational power needed to perform the simulations, and further increase the quantity of input data.

The paper is organized as follows. Section 2 presents the system studied and the roles that each agent can take. Five possibilities of interaction models are described in Section 3. The agents of the system are described in Section 4. This section refers extensively to Section 8 containing the optimization problems characterizing the behavior of the agents. Section 5 describes the implementation of the testbed as a simulator, an instance generator and a user interface. Section 6 discusses the simulation results, and Section 7 concludes.

\section{General view of the system}

Our analysis is centered on the short term operation of the medium-voltage (MV) network. In this time frame, active network management (ANM) consists in the coordination for the usage of flexibility to operate the distribution 


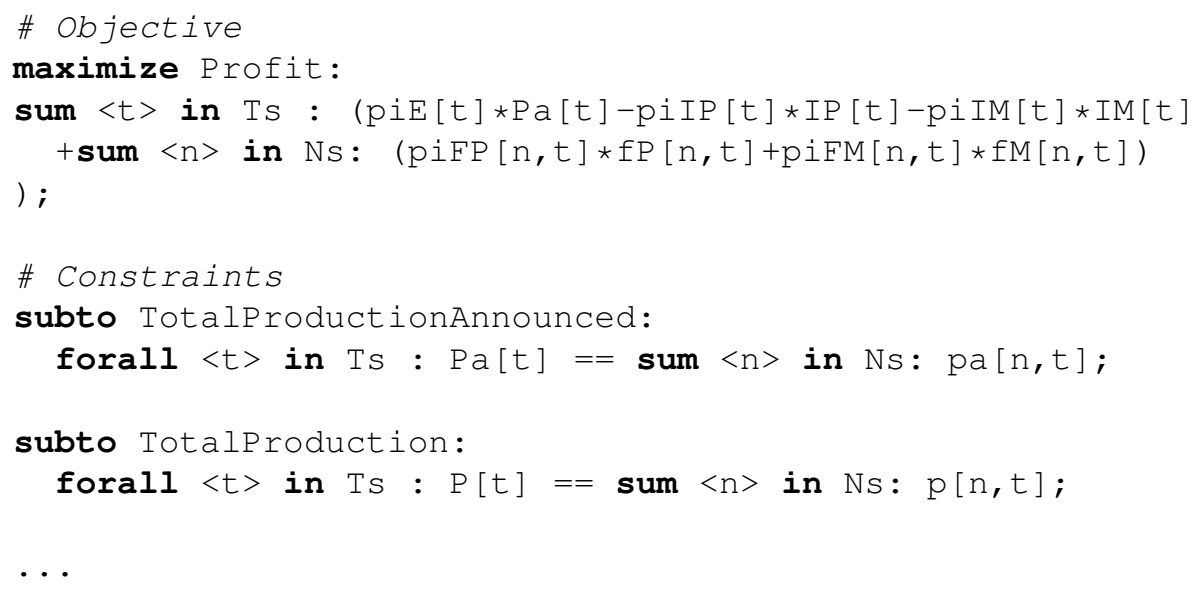

Figure 1: ZIMPL code of one optimization problem solved by a retailer.

system [27]. In the scope of this paper, we consider the short-term flexibility exchanges in an operational planning phase. These exchanges are governed by the interaction model that the agents must follow.

In the sequel of this document, we make the distinction between the roles and the actors. The roles considered in this paper are balance responsible parties (BRP), flexibility services providers (FSP) and flexibility services users (FSU). The actors simulated in this paper are the DSO, the TSO, producers and retailers, and may fulfill more than one role, depending on the interaction model. To operate its network, the DSO may act as a FSU. The TSO is, from the point of view of this simulation, a FSU with given needs of flexibility services. Producers and retailers both act simultaneously as BRPs, FSPs and FSUs.

All the interaction models we consider follow the procedure below.

1. DSO analysis and TSO imbalance settlement are based on reference baselines for every bus given by the BRPs.

2. On the day ahead, after the clearing of day-ahead energy market, BRPs submit their baselines to the DSO and the TSO.

3. The DSO assesses the state of the system and announces its flexibility needs to the FSPs. Other FSUs also announce their needs to the FSPs.

4. FSPs provide flexibility offers sequentially to each potential FSU, the first FSU being the DSO.

5. FSUs request and/or buy some proposed flexibility offers for their needs.

6. Closer to real-time, FSUs request the activation of their flexibility services.

7. Right before real-time, each FSP optimizes its realization taking into account the request of its FSUs, the penalty if a flexibility service is not 
provided and its imbalance with respect to the BRPs of the loads it controls.

8. The distribution network is operated using these realizations taking last resort actions if necessary, such as shedding buses. If such actions are needed and FSPs did not provide their service to the DSO, the FSPs are penalized at a regulated price.

We assume that there is an access contract between a BRP $a$ and the DSO for each bus $n$ the BRP has access to. A contract specifies a full access range $\left[l_{a, n}, L_{a, n}\right]$ and a wider flexible access range $\left[k_{a, n}, K_{a, n}\right]$, cf. Fig. 2. The BRP can produce or consume without any restriction within the full access range $\left[l_{a, n}, L_{a, n}\right]$, but in the flexibility intervals $\left[k_{a, n}, l_{a, n}\right]$ and $\left[L_{a, n}, K_{a, n}\right]$ the DSO can order a restriction of the production or consumption if necessary. In general, stakeholders are free to exchange flexibility services among them, but if the profile of a BRP is within the flexibility intervals, it has to propose flexibility offers to the DSO via FSPs.

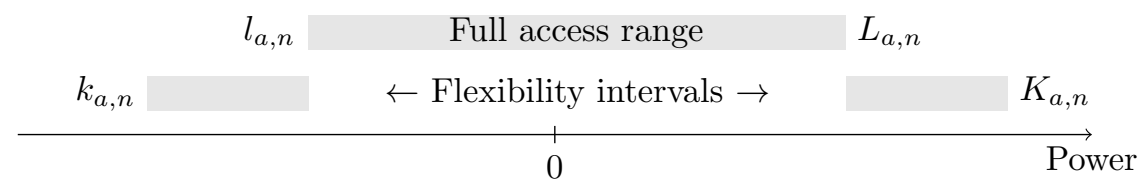

Figure 2: Definition of access bounds.

The following subsections detail the information exchanged and action taken by an actor with a given role. The index $a$ is used for an agent, $n$ for a bus and $t$ for a period. The set of buses to which an agent $a$ considered by an agent is denoted $\mathcal{N}_{a}$ and is a subset of the total set of buses $\mathcal{N}$.

\subsection{Balance Responsible Party}

Each BRP is responsible of its local and global baseline and performs the following actions:

1. Submit its global baseline to the TSO.

2. Submit its local baselines to the DSO.

3. Notify FSPs of the obligation to provide flexibility services if the local baseline is not in the full access range.

4. Pay the TSO for its global imbalance.

5. Pay the DSO for the local imbalance if a FSP for which the BRP is responsible did not provide a flexibility service.

The local baseline, $p_{a, n, t}^{b}$, which is positive for a production and negative for a consumption, is provided on a day-ahead basis by the BRP $a$ for each MV bus $n$ and each period $t$ to the DSO. The global baseline $P_{a, t}^{b}$ is provided by the 
BRP to the TSO. For the sake of clarity and conciseness, we consider that the $\mathrm{BRP}$ has only assets in the simulated system such that $P_{a, t}^{b}=\sum_{n \in \mathcal{N}_{a}} p_{a, n, t}^{b}$.

The global imbalance of the BRP, $I_{a, t}$, is computed considering the actual realization $P_{a, t}$, the total amount of flexibility activated $U_{a, t}$ and the flexibility provided $H_{a, t}$ :

$$
I_{a, t}=P_{a, t}-\left(P_{a, t}^{b}+U_{a, t}+H_{a, t}\right) .
$$

The imbalance cost to the BRP is given by

$$
\sum_{t \in \mathcal{T}}\left(\pi_{t}^{I^{+}} I_{a, t}^{+}+\pi_{t}^{I^{-}} I_{a, t}^{-}\right)
$$

where $I_{a, t}^{+}$and $I_{a, t}^{-}$are equal, respectively, to the positive and negative part of the imbalance in period $t, I_{a, t}$.

If the local baseline of the BRP, $p_{a, n, t}^{b}$ is within a flexibility interval such that $k_{a, n} \leq p_{a, n, t}^{b}<l_{a, n}$ or $L_{a, n}<p_{a, n, t}^{b} \leq K_{a, n}$, the BRP notifies the concerned FSPs that they must provide flexibility services such that the realization $p_{a, n, t}$ can be included in the full access range $\left[l_{a, n}, L_{a, n}\right]$. Note that in order to ensure that the FSP can propose such a service, it probably has to optimize itself the whole baseline $\left\{p_{a, n, t} \mid \forall t \in \mathcal{T}\right\}$.

The local information of the day-ahead baseline $p_{a, n, t}^{b}$ is used by the DSO to operate its system. If requests to activate flexibility are not satisfied, the BRP responsible for these FSPs is penalized at a regulated price proportional to the volume $\pi^{n}$ :

$$
\sum_{t \in \mathcal{T}} \sum_{n \in \mathcal{N}_{a}} \pi^{n}\left|\left(p_{a, n, t}^{b}+u_{a, n, t}+h_{a, n, t}\right)-p_{a, n, t}\right| .
$$

where $p_{a, n, t}$ is the local realization, $u_{a, n, t}$ the local flexibility activated in period $t$ and $h_{a, n, t}$ the local flexibility provided.

\subsection{Flexibility Services Provider and User}

The main interactions that the FSP conducts are:

1. Obtain the flexibility needs of the FSUs, the baselines and the obligations from the BRPs of the assets it controls.

2. Propose flexibility services.

3. Activate flexibility upon request of the FSUs.

Flexibility services need a reference to be quantified. In this paper we chose a baseline $p_{a, n, t}$ provided by the BRP. Note that if the FSP is its own BRP, the agent with the combined roles can optimize its baseline to maximize the flexibility it provides. The other data at the FSP disposal is an indicator of the flexibility needs in each bus and each period upward and downward. The latter indicator is provided by the FSUs to help the FSP to quantify the action it is able to perform.

A FSP offers a service $i$ at a reservation price $\pi_{i}^{r}$ and an activation price $\pi_{i}^{b}$. We consider two types of flexibility services which are represented in Figure 3 : 
a single period flexibility service, tailored for instance for curtailment offers of wind generation units, which defines a modulation range $\left[m_{i, \tau_{i}}, M_{i, \tau_{i}}\right]$ available for a given period $\tau_{i}$; and energy constrained flexibility service which defines for each period $t$ a modulation range $\left[m_{i, t}, M_{i, t}\right]$. A user of the latter service may choose the modulation for each period under the constraint that the average modulation is zero. This service is well tailored for load modulation because the energy procured to the load is preserved over the time horizon with respect to the baseline consumption.

In the last part, FSUs that have contracted some services provide their activation requests to the FSPs. The total desired modulation at bus $n$ and period $t$ is denoted by $h_{a, n, t}$. The FSP controls its assets to provide the desired modulation with respect to the precomputed baseline.

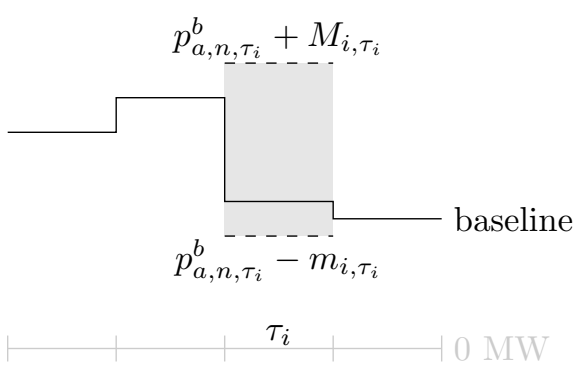

(a) Single period

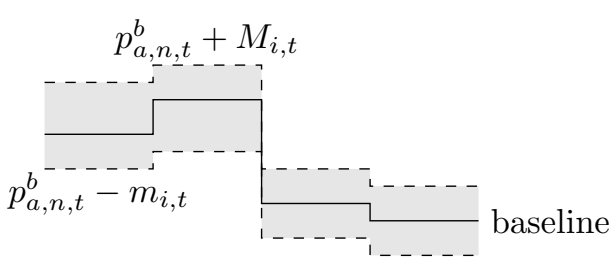

(b) Energy constrained

Figure 3: Flexibility services implemented.

A FSU has three main interactions with the system.

1. Compute and communicate an indicator of its upward and downward flexibility needs in adjustable power for each bus and each period.

2. Evaluate the flexibility offers from the FSP and select the services to contract.

3. Request the activation of the contracted flexibility services.

How the needs are defined, the offers are evaluated and the activation requested depends on the agent acting as FSU, cf. Section 4. For instance, the DSO computes its flexibility needs to overcome anticipated congestions.

\section{Interaction models}

The differences between interaction models originate from the usage the stakeholders make of flexibility services, from the remuneration and financial compensations associated to the flexibility services, and from the terms of access contracts delivered to the grid users. Access contracts define whether and 
to which extent the DSO could require flexibility from its grid-users. We consider that a FSP first makes a request to the DSO to get access to a range $\left[g_{a, n}, G_{a, n}\right]: g_{a, n} \leq 0 \leq G_{a, n}$. Based on these requested access bounds, the DSO computes safe access bounds $\left[b_{a, n}, B_{a, n}\right]: b_{a, n} \leq 0 \leq B_{a, n}$. The safe access range ensures that no congestion occurs if every grid user accesses to the network in the limits $\left[b_{a, n}, B_{a, n}\right]$. These bounds are represented in Figure 4. A more comprehensive definition of this procedure is outside the scope of this paper. Section 4.1 presents one simple method adopted for the simulation.

Given the previous definitions, the five proposed interaction models governing flexibility exchanges with the DSO are detailed below. Stakeholders are free to exchange flexibility services among them, but in Models 3-5, if the profile of a BRP is within the flexibility intervals, it has to propose flexibility offers to the DSO via FSPs.

Model 1. The DSO does not use any flexibility service and does not restrict grid users: $\left[l_{a, n}, L_{a, n}\right]=\left[k_{a, n}, K_{a, n}\right]=\left[g_{a, n}, G_{a, n}\right]$.

Model 2. The DSO does not use any flexibility service and allows grid users to produce or consume only in the safe access bounds: $\left[l_{a, n}, L_{a, n}\right]=\left[k_{a, n}, K_{a, n}\right]=$ $\left[b_{a, n}, B_{a, n}\right]$.

Model 3. The DSO sets $\left[l_{a, n}, L_{a, n}\right]=\left[b_{a, n}, B_{a, n}\right]$ for each agent but allows access in the range $\left[k_{a, n}, K_{a, n}\right]=\left[g_{a, n}, G_{a, n}\right]$. The grid users may be restrained to keep their production/consumption into the range $\left[l_{a, n}, L_{a, n}\right]$ upon request of the DSO. This restriction does not lead to financial compensation by the DSO except for the imbalance created by the request.

Model 4. This model is equivalent to Model 3 but the DSO pays for the activation of the flexibility of the grid users. For instance, this case allows producers to recover from the loss of the subsidies for green energy generation.

Model 5. The DSO does not oblige grid-users to provide flexibility, i.e. $\left[k_{a, n}, K_{a, n}\right]=$ $\left[l_{a, n}, L_{a, n}\right]=\left[g_{a, n}, G_{a, n}\right]$. The DSO can however contract flexibility like any FSU.

The purpose of this paper is thus to provide a detailed explanation of the platform to analyze the proposed interaction models, but it also enables the definition of other interaction models and carrying on more analyses. New interaction models can be easily defined using the following six parameters. More advanced modification can be carried out by modifying the open-source code.

1. Is the DSO a FSU? Define if the DSO uses flexibility services. The value is false for Models 1-2 and true for Models 3-5.

2. What is the cost at which the DSO buys flexibility? Different options are implemented in this testbed. First, the DSO may pay the same cost that a TSO or a BRP would pay. Another alternative is that the DSO only pays for the imbalance caused by the activation of the flexibility. A last option 


\begin{tabular}{ccc}
$g_{a, n}$ & Requested range & $G_{a, n}$ \\
& Safe range & $B_{a, n}$ \\
\hline$b_{a, n}$ & + & Power
\end{tabular}

(a) Base data

$\begin{array}{lll}l_{a, n} & \text { Full access range } & L_{a, n}\end{array}$

(b) Unrestricted access. Models 1 and 5

$l_{a, n} \quad$ Full access range $\quad L_{a, n}$

(c) Restrictive access. Model 2

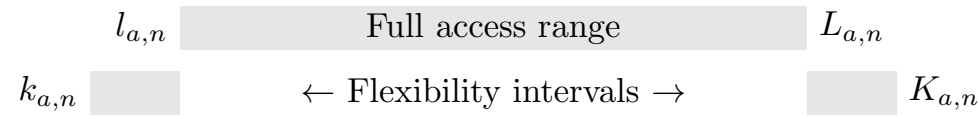

(d) Flexible acces. Models 3 and 4

Figure 4: Definition of the access bounds for each interaction model.

defines lower costs for downward modulation services. These alternative costs are used in Models 1-5. The activation cost of downward modulation flexibility services is set to the energy price in the corresponding period minus the marginal cost of the producer, which is approximately the benefits of the producer. Indeed, at the stage of flexibility offers, the producer has already sold its energy and as the distribution network has not enough capacity to carry the energy, the DSO must pay the imbalance tariff by the activation of flexibility services. In this setting, the benefits of the producer are attributed to the DSO to reduces these imbalance costs.

3. What is the imbalance price paid by the DSO compared to BRPs? In all interaction models described previously, the imbalance tariff paid by the DSO is the same as any BRP. A preferential tariff could be applied to the DSO as it can notify the TSO in advance of the flexibility services activated and therefore the resulting imbalance.

4. Does the DSO restrict the grid users? Three restrictions pattern are implemented: restrictive, flexible and unrestricted access. These patterns are represented in Figure 4.

5. What is the frequency of the access bound computation? Prior to the day by day simulation of the system, the DSO computes the access bounds $\left[b_{a, n}, B_{a_{n}}\right]$ to its system. If every actor of the system access to the network within these bounds, no further actions needs to be taken by the DSO to ensure the secure operation of its network. In a real system, 
this procedure would not be performed in one round, since access contracts are delivered within a certain delay after they were requested. The aggregation of contracts of the low voltage contributors should also be considered. A more realistic simulation would thus consider the legacy of access contracts. In the framework of this simulation, two possibilities are available: a computation based on the installed capacity and one based on the maximum values observed by the grid user on the time horizon of the simulation. The first option is more realistic but the second might be considered in the future to increase the freedom of the grid users. In Models 1-5, the option chosen is the computation based on the installed capacity.

\section{Agents}

The agents simulated in this paper are the DSO, the TSO, producers and retailers. They may fulfill more than one role depending on the interaction model. To operate its network, the DSO may act as a FSU, as in Model 5 for instance. The TSO is, from the point of view of this simulation, a FSU with given needs of flexibility services. Producers and retailers both act simultaneously as BRPs, FSPs and FSUs. The complete optimization problems solved by the agents are provided in the Appendix. The global interaction between the actors are summarized in Figure 5. The courses of the events is described from left to right going from short-term interactions to settlement. Each type of actor is represented by a horizontal line. Each vertical arrow represents an interaction between two types of actor. The actor on the head of the arrow receives the information from the ones at the tails of the arrow indicated by the circles.

\subsection{Distribution System Operator}

In our simulations, the DSO acts with the objective to reach the technical optimum of the system. Prior to the day by day simulation of the system, the DSO computes the access bounds $\left[b_{a, n}, B_{a_{n}}\right]$ to its system. If every actor of the system access to the network within these bounds, no further actions needs to be taken by the DSO to ensure the secure operation of its network. These bounds are obtained by solving optimization problem (5).

Next the DSO acts as a FSU. Taking as input the baselines provided by the BRPs and the characteristics of the network, the DSO provides an indicator of its flexibility needs in MW for each bus and each period so as to alleviate congestion in the lines. These needs are obtained by solving problem (6).

For the flexibility procurement, the DSO solves problem (7). Note that even though the DSO is not a BRP, it pays imbalance fees caused by its usage of flexibility. This incentivizes the DSO to activate, when it uses one flexibility service downward to alleviate a congestion, another one upward to restore the balance.

The flexibility activation optimization problem is identical to (7) except that it considers only the contracted flexibility services and their activation costs. 
Producers

\& Retailers

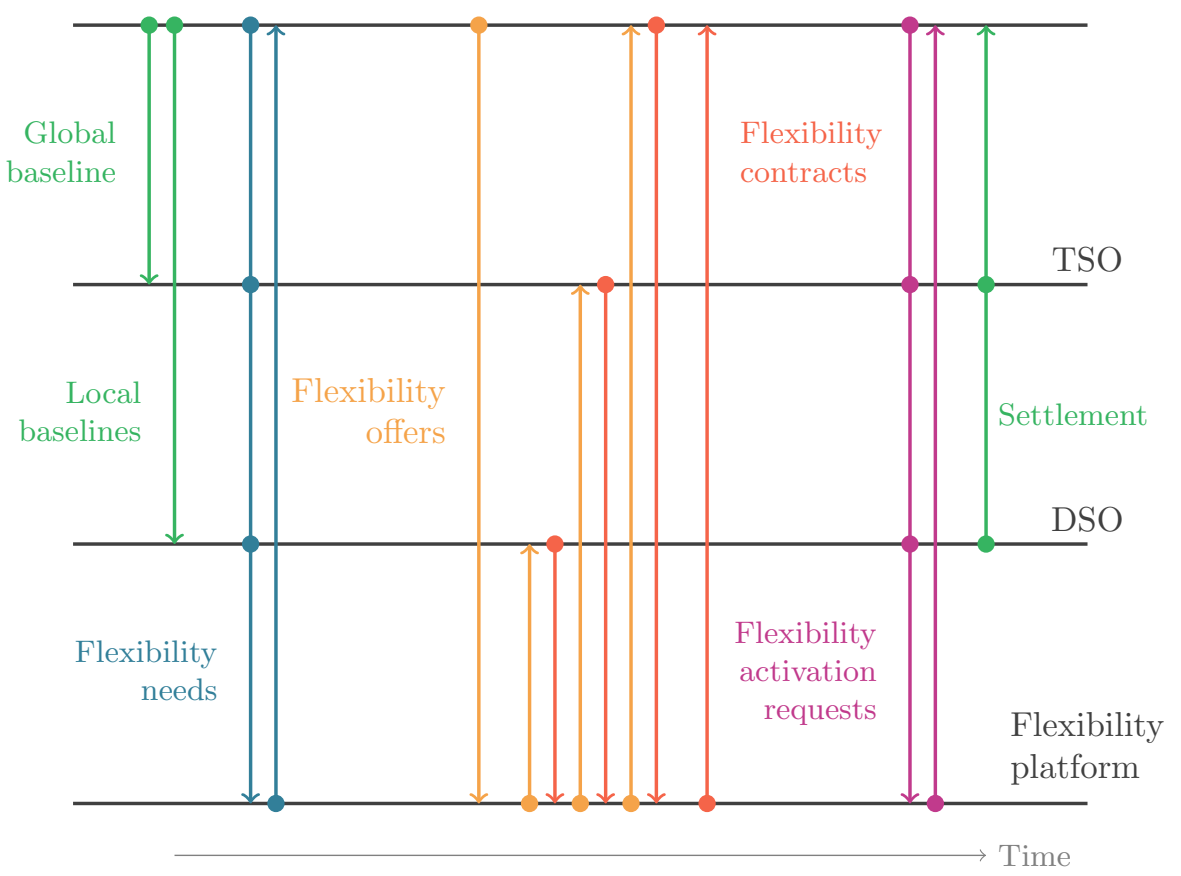

Figure 5: Timeline of the interaction between the agents.

Note that as we use a deterministic simulation, nothing changes between the reservation phase and the activation phase. As a result, the decisions taken in the activation phase are identical to the ones foreseen in the reservation phase.

Finally, we opt for a simple real-time system control strategy based on the realizations of the agents, which mimics a protection scheme. If the flow in some lines is over their thermal capacity, the DSO sheds production or consumption according to optimization problem (8).

\subsection{Producer and retailer}

A producer is a BRP, a FSU and a FSP. In order, a producer announces its baselines, requests flexibility services, offers flexibility services, buys and activates flexibility services, and finally regulates its balance. A retailer is an actor which retails energy to its customers and manages aggregated loads at the MV level. It fulfills the same roles as the producer and therefore follows exactly the same steps. For the sake of conciseness, we detail only the producer. The specific optimization problems of the retailer (13), (14), (16) and (17), are given in Section 8.

The baselines of the producer are obtained by solving optimization problem (9). Already in this stage, the producer considers the possibility to be in 
imbalance considering the expected imbalance price. The problem takes as parameters the total upward and downward flexibility needs for each bus and each period. These parameters could come from forecasts of the producer or FSUs. The first approach is adopted in this paper, where the forecast is based on the flexibility needs communicated by the FSU in the previous days. Note that the real needs of the flexibility users are communicated after that the baselines are obtained according to the interaction models.

Once the producer is notified of the acceptance of its offers on the day-ahead energy market, it optimizes the flexibility it is able to provide with respect to the announced baseline. This step is modeled by optimization problem (10). The producer procures and activates flexibility services based on the solution of problem (11).

Finally, the producer decides on the actual realizations for each bus and each period by solving problem (12) considering as parameter the flexibility activation requests as a FSP and its flexibility requests as a FSU. The producer minimizes its imbalance in each period and also ensures that the realization in the bus in which it should deliver a flexibility service is equal to the expected value. The local imbalance is penalized at a regulated price.

\subsection{Transmission System Operator}

The TSO is a pure FSU in this simulation. Its flexibility needs are taken as data and are not localized. Any FSP may in principle provide flexibility offers to the TSO. The flexibility contracted by the TSO is computed by optimization problem (18). As the TSO can procure flexibility outside the distribution system, the surplus of this stage is taken as the amount of flexibility collected in this network weighted by a price representing the system benefits of contracting reserve into the considered distribution network.

When the TSO decides to activate flexibility services, the TSO solves an imbalance external to the considered distribution system. Note that imbalance coming from the considered distribution system should not be solved by local flexibility services to avoid counterbalancing DSO actions. The benefits of the TSO coming from the activation of flexibility services in this part of the network are computed by optimization problem (19).

\subsection{Flexibility platform}

The flexibility platform is an intermediary between FSUs and FSPs. This platform could be operated either by a DSO, a TSO or an independent party. One could implement interaction models without a flexibility platform where FSUs and FSPs exchange directly their services. The flexibility platform facilitates the interaction and can anonymize the flexibility exchanges. Note that a pure market based platform would probably not be a good alternative due to the lack of liquidity as these services are highly dependent on the location.

In our settings, the flexibility platform collects the flexibility needs of the FSUs and communicates them to the FSPs. FSPs submit their offers to the platform which sequentially proposes them to the DSO, to the TSO and finally 
to other FSUs as depicted in Figure 5. As the DSO solves local problems such as congestions, its needs can be satisfied by fewer services than imbalance problem faced by the TSO or BRPs. This motivates the priority given to the DSO, but other rankings could be investigated.

\section{Implementation}

The testbed is divided in three parts: the simulator, an instance generator and a user interface. The first two are implemented in Python 3. The user interface is composed of a client in HTML5-Javascript and a server in Python 3 which facilitates the use of the generator and the simulator, and provides an interface to visualize the results.

\subsection{Simulator}

The simulator takes as input the parameters of all agents on the simulated horizon, i.e. one day. A one year simulation can be performed by simulating independently each day. All the parameters of the optimization problems given in Section 8 should be provided by CSV files. A self detailed example is given in the implementation. An additional CSV file contains the parameter of the interaction model which are listed in Table 1. The specific combination of the parameters of Table 1 to obtain the models of Section 3 are encoded in the user interface of Section 5.3.

\begin{tabular}{ll} 
Parameter & Description \\
\hline DSOIsFSU & DSO is a FSU. \\
& Value: True or False. \\
DSOFlexCost & Sets the cost at which the DSO buys flexibility. \\
& Value: imbalance, normal or full. \\
DSOImbalancePriceRatio & Ratio of the imbalance price that is paid by the \\
& DSO with respect to the one paid by BRPs. \\
& Value: Number, default value is 1.0. \\
accessRestriction & Restricted access to the DSO network follow- \\
& ing the access agreement step of the DSO. \\
& Value: none, conservative or flexible \\
accessBoundsComputation & Access bounds computation type. \\
& Value: horizon or installed.
\end{tabular}

Table 1: Parameters of an interaction model.

Based on all the parameters, the simulator performs the simulation of one time horizon, e.g. one day. The simulation requires solving the optimization problems of the actors, which are encoded in ZIMPL and solved using the SCIP solver [28]. To conduct the simulation, some agents need predictions of some parameters such as the flexibility needs of the FSU (see Section 4.2 for more details). The simulation is initiated with these predictions set to zero. If the 
realizations does not match the predictions after one run, the simulation is reinitialized with the new realization. This process is repeated until the realizations match the predictions or after a predetermined number of iterations.

\subsection{Instance generator}

The instance generator allows a user to create an instance from high-level parameters instead of having to specify manually the assets of each agents. The reduced set of parameters is given in Table 2 to which we add the interaction model parameters of Table 1 .

\begin{tabular}{ll} 
Parameter & Type \\
\hline Title of the instance & String \\
Network & Network name, see Figure 6 \\
Total production & Mean and maximum value in MW \\
Production cost & Price in $€ /$ MWh \\
Total consumption & Mean and maximum value in MW \\
Retailing price & Price in $€ / M W h$ \\
Energy price & Mean and maximum value in \\
& $€ /$ MWh \\
Days of simulation & Start and end day in $\{1, \ldots, 365\}$ \\
Interaction model parameters & See Table 1 \\
& \\
Number of periods by day & Integer \\
Number of producers and retailers & Integer \\
External imbalances of the producers & Percentage of their total volume \\
and retailers & \\
Retailers flexibility reservation cost & Price in $€ / M W h$ \\
TSO flexibility needs & Volume in MW \\
TSO reservation price & Price in $€ / M W h$
\end{tabular}

Table 2: Parameters of the instance generator.

The instance generator takes as basis a network. The network already integrated are given in Figure 6. Along with their topology is given for each bus the ratio of installed production and consumption with respect to the one of the whole network. We now detail the generation of the CSV parameter files for the simulator.

Generation of the producers. The producers are generated from data of a wind farm in 2013 scaled to the input mean and maximum production in the network. Each bus with production in the selected network is assigned randomly to one producer. The maximum production for each period is set to the scaled baseline value times the contribution of the bus with respect to the total production in the network. The minimum production is the latter value minus the flexibility of the producer. The external imbalance of the producer is generated uniformly between plus and minus its mean production. 


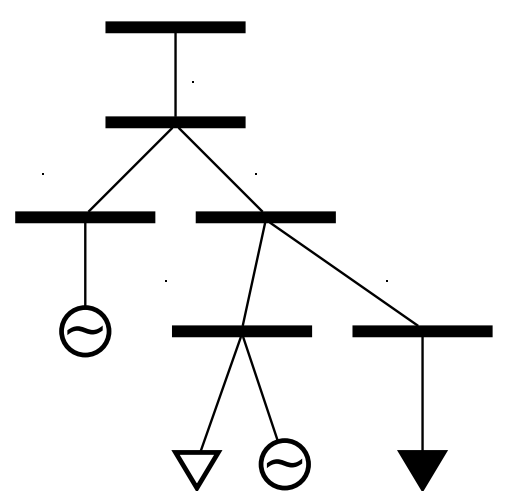

(a) Six buses example network.

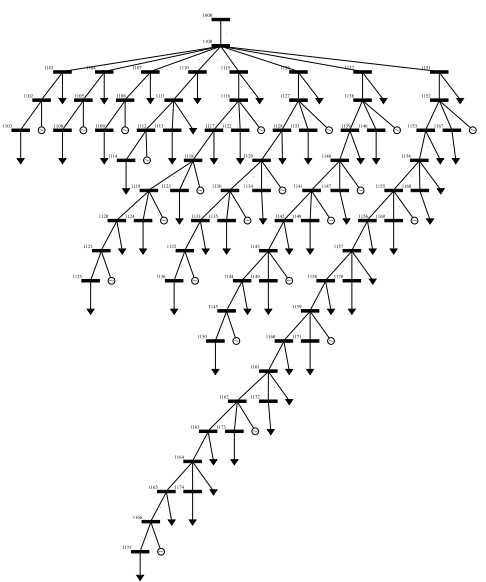

(b) 75 bus network from [29].

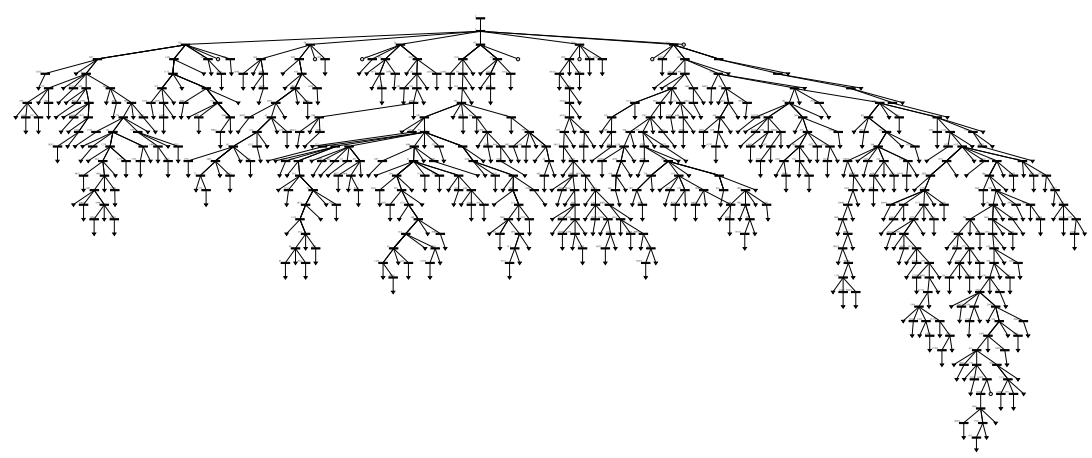

(c) Ylpic network.

Figure 6: Networks available.

Generation of the retailers. The consumption curve is built from the Belgian total load consumption of 2013 [30]. The share of each retailer is randomly generated so that the shares sums up to one. The mean baseline consumption of a retailer in one bus is the total consumption curve times the retailer's share and the ratio of the consumption with respect to the one of the total network. The sum on each period of the horizon of mean consumption provides the total energy to consume $V_{a, n}$. The minimum and maximum realization bounds, $p_{a, n, t}^{\min }$ and $p_{a, n, t}^{\max }$, are given by the mean consumption plus and minus the consumption flexibility.

Generation of the price curves. The prices are generated by scaling concurrently the Belpex day-ahead energy market prices [31] and the Belgian imbalance tariffs of 2013 [30]. To ensure the coherence of the input parameters of the simulator, the generator sets the minimum imbalance tariff to the energy prices. If it was 
not the case, an agent could sell energy to the market without producing and still get benefits from this controversial action.

Generation of the TSO. The flexibility needs of the TSO, $R_{t}^{-}$and $R_{t}^{+}$, are constant values given as input parameters. The external imbalance that the TSO faces in each period is randomly generated following a uniform distribution between $-R_{t}^{-}$and $R_{t}^{+}$.

Generation of the flexibility potential indicators. To operate its system, the DSO needs indication on where flexibility can be obtained in its network. This information could for instance come from access contracts or notification from the FSUs willing to sell their services to the DSO. In this testbed, this knowledge of potential availability of flexibility services is taken as parameter in optimization problem (6) which allows the DSO to communicate its flexibility needs to the FSU based on the baselines of the BRPs. The indicators of upward and downward flexibility availability for each bus, respectively $\alpha_{n}^{+}$and $\alpha_{n}^{-} \in[0,+\infty[$ are dimensionless parameters. A 0 indicator indicates than no flexibility is available while a large value indicates that a large amount of flexibility can potentially be contracted in the bus with respect to the others.

In the instance generator, these indicators are arbitrarly computed by the following procedure. First, the generator computes the installed flexible production and consumption in each bus in terms of power. We denote them respectively $\kappa^{+} \in\left[0,+\infty\left[\mathrm{MW}\right.\right.$ and $\left.\left.\kappa^{-} \in\right]-\infty, 0\right]$ MW. Second, these data are used to define the indicators:

$$
\begin{aligned}
\alpha_{n}^{+} & =\frac{1}{\kappa^{+} / 10-\kappa^{-} / 10} \\
\alpha_{n}^{-} & =\frac{1}{\kappa^{+} / 1-\kappa^{-} / 10}
\end{aligned}
$$

where the numeric coefficient gives more importance to downward flexibility by the production side.

\subsection{User Interface}

The user interface simplifies the access to the instance generator described in Section 5.2 and the simulator of Section 5.1. Screenshots of the interface are given in Figure 7. The home screen, shown in Figure 7a, allows the creation of an instance via the generator giving it parameters such as the interaction model or the total production in the network. The global results screen, seen in Figure $7 \mathrm{~b}$, provides a summary of the simulation results of an instance and graphs to see the evolution of the welfare or DSO costs day by day. Daily results can be seen in the screen of Figure 7c. The state of the network in each period is represented and specific results to each bus and line can be obtained by clicking on it in the network picture.

The webpage includes a Javascript client communicating with a Python server which can either be on the same computer or a different one. The server 


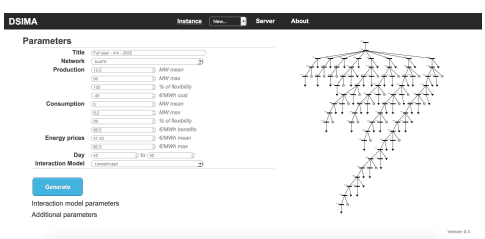

(a) Homepage with the parameters of the instance.

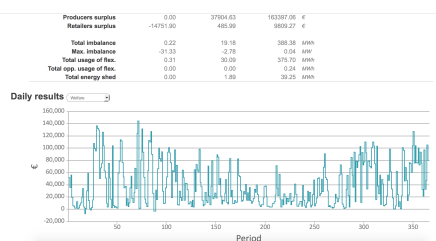

(b) Global results for the simulated horizon.

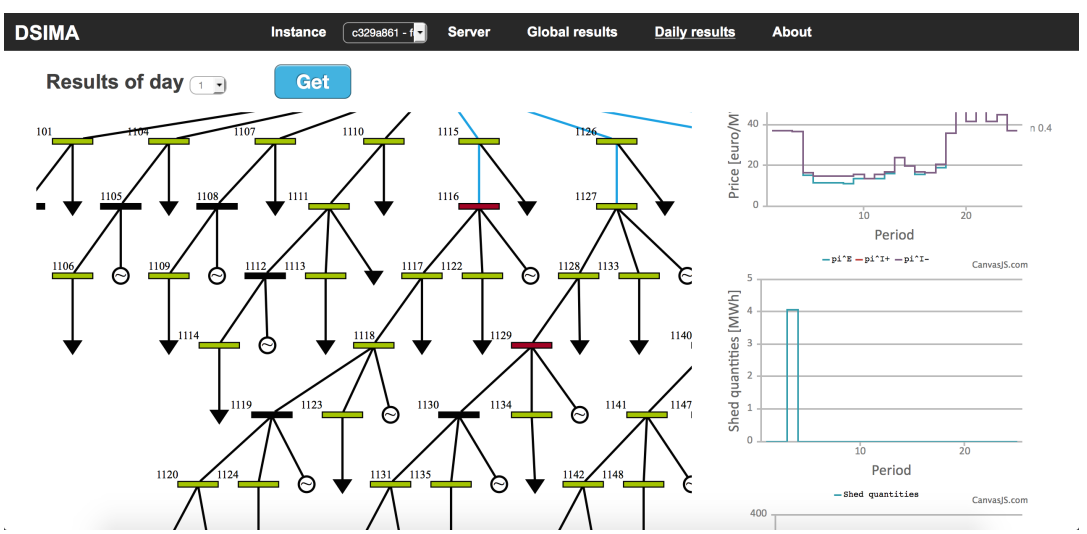

(c) Daily results. Each bus and line can be clicked on to display its specific informations.

Figure 7: Screenshots of the user interface.

handles the requests of the user and executes the instance generator or simulator. Note that, the server can handle multiple clients at the same time, queuing the simulation requests if needed. The simulations of multiple days is performed efficiently by assigning the simulation of each day to a different thread.

\section{Results}

The test case for the results is described in Section 6.1. A typical one-day run is presented for one interaction model in Section 6.2. Finally, the comparison of the five interaction models over one year is presented in Section 6.3. The experiments are carried out on a computer equipped with an Intel Core i7-3770 $\mathrm{CPU}$ at $3.40 \mathrm{GHz}$ with $32 \mathrm{~GB}$ of RAM. The optimization problems are solved with SCIP [28].

\subsection{Test case}

The interaction models are tested on a generic $11 \mathrm{kV}$ distribution network composed of 75 buses and hosting 22 distributed generation units [29]. The network topology is fixed. The simulation is run for a time horizon of one day 
divided in 24 periods. Production data of the 22 generation units are taken from a production curve of 2013 scaled such that the maximum production reaches $64.2 \mathrm{MW}$ and the mean production is $16 \mathrm{MW}$. Distributed generation units are clustered into three portfolios, each managed by a different producer. The producers only ask for a remuneration of the activation of their flexibility services. The consumption of the 53 connected loads is built from the Belgian total load consumption of 2013 scaled to a mean of $10.8 \mathrm{MW}$ and a maximum of $21 \mathrm{MW}$ [30]. This consumption is divided in three parts, each belonging to one retailer. The consumption of a retailer is divided in a static part and a flexible part respectively accounting for $80 \%$ and $20 \%$ of the total consumption of the load. The retailer proposes its flexibility with no activation fee but requires a reservation fee. The reservation fee of a demand side flexibility offer is assumed to be $5 € / \mathrm{MWh}$. The TSO flexibility aims to contract a volume of flexibility equal to $2 \%$ of the total installed production capacity of the system in each period. We use the following reservation prices of secondary reserve: $\pi_{t}^{S^{+}}=-\pi_{t}^{S^{-}}=45 € / \mathrm{MWh}$. The total activation request of the TSO is drawn using a zero mean Gaussian distribution with a variance equal to the target flexibility volume of the TSO. Energy prices are taken from the clearing of 2013 of the Belpex day-ahead energy market [31] scaled to a mean of 53.64€/MWh and a maximum of $93.03 € / \mathrm{MWh}$, excluding the extreme $2.5 \%$ of the original data. The value of lost load taken into account in the case of shedding due to the tripping of a protection is set to $500 € / \mathrm{MWh}$ for the production and $1000 € / M W h$ for the consumption. The imbalance prices come from the Belgian TSO [30]. A FSP not providing a contracted flexibility service or violating its dynamic range is penalized at $150 \%$ of the maximum imbalance price.

\subsection{Typical run}

We first provide illustrative results for Model 4 applied to the test system based on data of July 10, 2013. Figure 8a illustrates the events happening in the course of the simulation. Shedding an MV bus disconnects only the devices connected to that bus, and does not impact surrounding MV buses of the system. The maximum flow injected in the transmission network is $44.1 \mathrm{MW}$ at period 20. The DSO sheds a total of 12.6 of the $913.44 \mathrm{MWh}$ of generation potential, and activates $11.57 \mathrm{MWh}$ of flexibility, on a total of $17.8 \mathrm{MWh}$, which causes an equal imbalance volume. The total imbalance, i.e. the sum of the imbalances over all agents in the system, is $25.8 \mathrm{MWh}$. The total flexibility activated as well as the imbalance of all agents in the system is given in Fig. 8b. The total welfare of the system is $32107 €$. The total DSO cost is $823 €$ and does not consider remuneration for the shed quantities. However, the value of lost load and production are taken into account in the welfare value. The total benefit of all retailers is $3340 €$. Benefits earned by the producers sums up to $29455 €$. The TSO obtains a welfare of $1400 €$ from the services acquired in the system. A one day simulation lasts 312 seconds. 


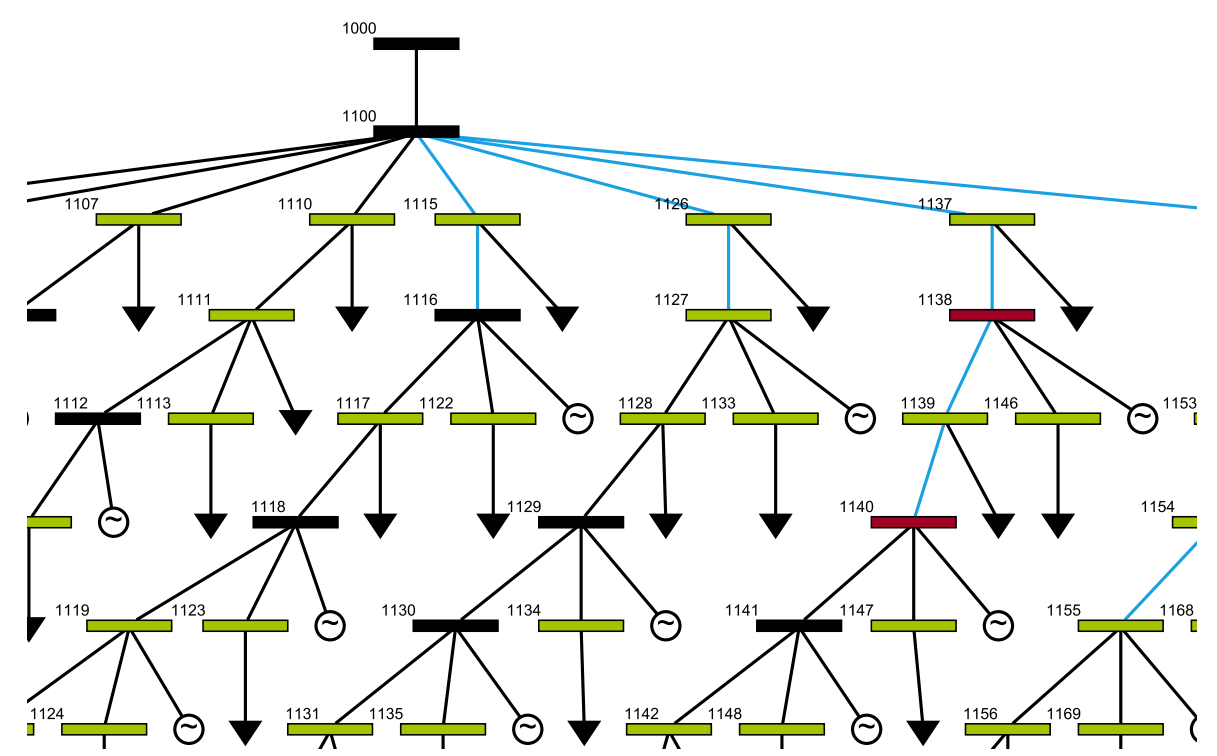

(a) A bus is colored in green if flexibility is used locally for at least one period of the simulation horizon. Similarly a bus is colored in red when shedding occurs, and a line is colored in blue when a thermal limit is reached.

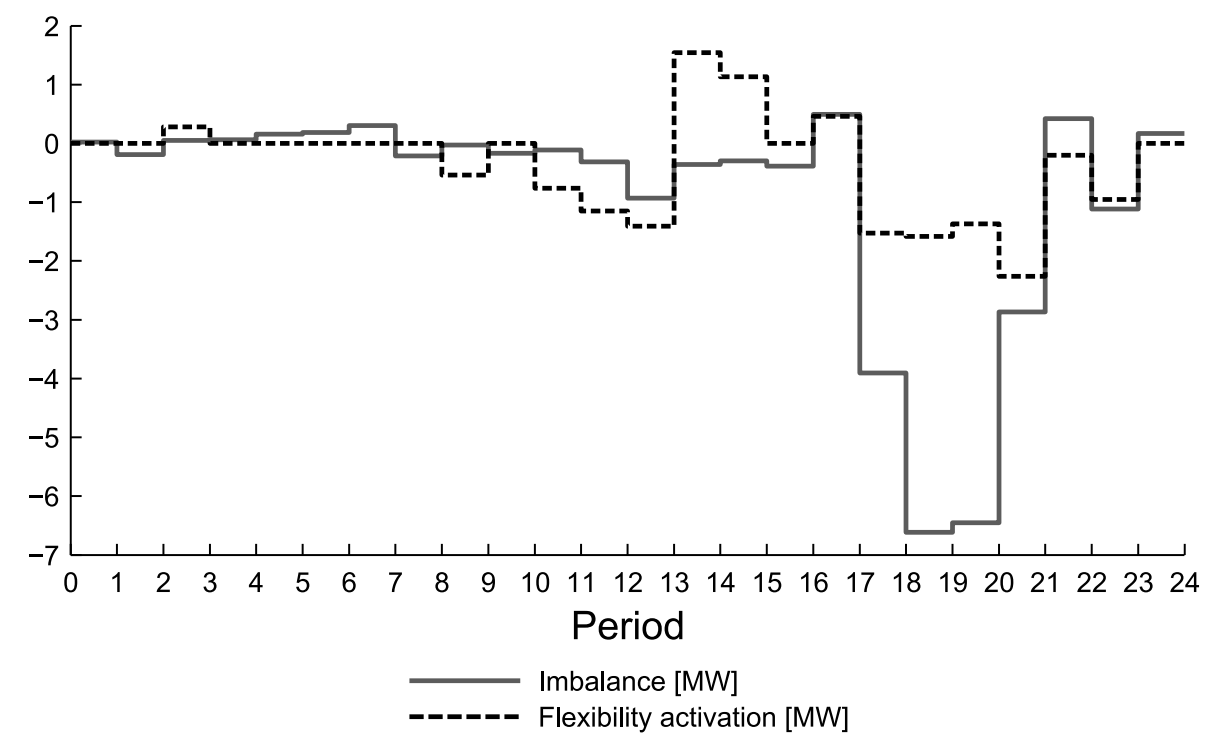

(b) Total flexibility activated and imbalance for all agents in the system.

Figure 8: Typical run on the test system.

\subsection{Comparison of the interaction models}

All the models are simulated under the same conditions for 365 days individually, which amounts to 32 hours of simulation by model. The main results 
are reported in Table 3 .

Model 1 highlights that leaving the network with no control leads to high shedding and impacts the welfare with a penalty of about $12000 €$ by day. Only Model 2 yields no shedding but a lower welfare due to the conservative actions of the DSO to restrict the access of production units. This conservative strategy penalizes the producers which are not allowed to produce in situations where the network can handle higher injections. Using ANM strategies as it is proposed in Models 3, 4 and 5 leads to equivalent and higher welfares even with average shedding penalties of $1000 €$ per day. These models would be more efficient if shedding could be avoided. This necessity is caused by the activation of flexibility services by the TSO in an opposite direction to the directives of the DSO. The DSO activates flexibility services based on the baselines so that once the services are activated, no congestion occurs anymore. However, the actual realization differs from the expectation of the DSO as it is not the only user of use flexibility services. A better coordination between the DSO and other FSUs would lead to even higher welfare and is necessary to ensure the safety of the system on the long term.

Only the TSO uses demand side flexibility in this simulation. Even though flexibility services from the demand side are cheaper, their usage is expensive for the DSO which must compensate the imbalance created to solve a congestion problem. In addition, an activation of an energy constrained flexibility offer in one period requires another activation in a different period and therefore up to a double imbalance compensation. A variant of these interaction models could allow a discount for the DSO on the imbalance tariff.

Table 4 compares the surpluses of the producers in the last four interaction models with respect to the first one. These results show that Model 2 significantly decreases the surplus of each producer. The smallest producer is the most sensitive to the choice of the interaction model but still the difference between Models 3, 4 and 5 on its surplus is of the order of $1 \%$. The two other producers are less impacted by the ANM interaction models. This result may motivate producers to bargain their flexibility for free, as long as the imbalance is paid, in order to obtain an increased access to the distribution network.

\section{Conclusion}

This paper proposes the open-source testbed DSIMA built to analyze quantitatively interaction models for the exchange of flexibility within distribution networks. The testbed simulates the interactions of the DSO, the TSO, producers and retailers in a medium-voltage distribution network. The simulation is performed in an agent based framework where each agent maximizes its individual objective. The testbed is composed of an instance generator, a simulator and a web-based user interface written in Python and HTML5/Javascript. These three modules allow various compromises between flexibility and ease of modification.

This paper formalizes completely the interactions between the stakeholders of a distribution system and all the interaction models. Our analysis of the 


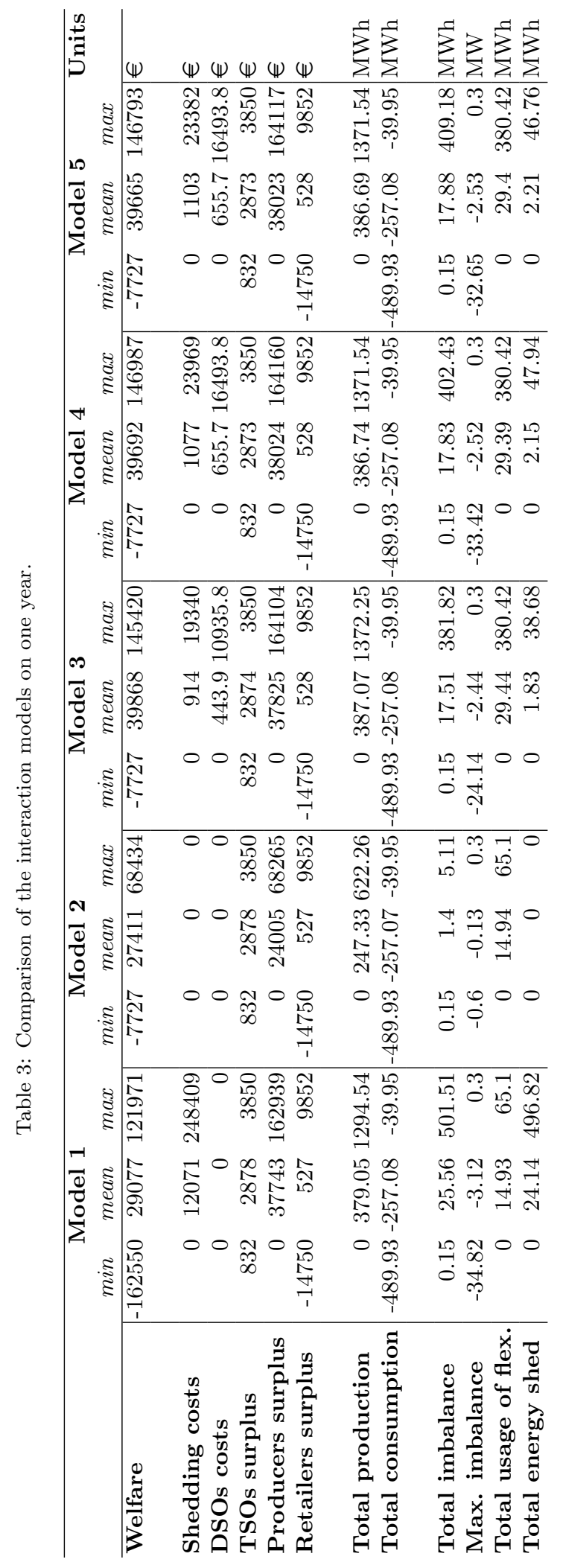


Table 4: Comparison of the annual surpluses of the producers with respect to Model 1.

\begin{tabular}{l|rrrrr|r}
\hline & Model 1 & Model 2 & Model 3 & Model 4 & \multicolumn{1}{c}{ Model 5 } \\
\hline Producer 1 & $20647 €$ & -34.97 & -0.09 & 0.43 & 0.52 & $\%$ \\
Producer 2 & $3287 €$ & -44.70 & 3.06 & 4.26 & 3.66 & $\%$ \\
Producer 3 & $13809 €$ & -36.56 & -0.01 & 0.38 & 0.37 & $\%$
\end{tabular}

simulation results tends to show that the active network management based interaction models lead to the larger welfare. However, these models, in their current version, cannot guarantee the operation of the network without shedding due to the lack of coordination between the DSO and the TSO. Restricting grid users to an access range computed ahead of time to prevent any congestion is the only option out of the proposed models to avoid shedding. The conservative interaction model is shown to penalize some producers more than others, raising the question of fairness of access range allocation and flexibility activation. However, results show that in the ANM models, producers may have incentive to bargain their flexibility for free, as long as the imbalance is paid, in order to obtain an increased access to the distribution network. Introducing a coordination mechanism to ensure a globally coherent activation of the flexibility would therefore lead to an interaction model with the largest welfare while avoiding shedding.

The work presented in this paper could be extended along several lines. In the proposed interaction models, the BRP is responsible of providing baselines for every medium-voltage bus. However several stakeholders may forecast these references: the DSO, FSPs, BRPs, etc. and these choices should be further investigated by implementing them in this testbed. Additional implementation could add new interaction models or flexibility services. One could also refine the modeling level, for instance considering alternating current power flow equations or alternative agent behaviors. The open-source code needed to continue this work can be found at the address http://www.montefiore.ulg.ac.be/ $\sim$ dsima/.

\section{Acknowledgments}

This research was supported by the public service of Wallonia - Department of Energy and Sustainable Building, within the framework of the GREDOR project.

\section{Optimization problems of the agents}

\subsection{Nomenclature}

In the following of the section the letters $a, i, t$ and $n$ index agents, flexibility services, time periods, and buses, respectively. Superscripts ${ }^{+}$and ${ }^{-}$depict an upward or a downward modulation. 
Parameters.

$\begin{array}{ll}\alpha_{n} & \text { Flexibility potential indicator } \\ \mathcal{A} & \text { Set of agents } \\ C_{n, m} & \text { Capacity of the line }(n, m) \\ c_{a, n, t} & \text { Marginal cost of producer units } \\ d_{n} & \text { Flexibility potential indicator } \\ E_{a, t} & \text { External imbalance to the system } \\ {\left[b_{a, n}, B_{a, n}\right]} & \text { Safe access bounds to the network } \\ {\left[g_{a, n}, G_{a, n}\right]} & \text { Requested access bounds to the network } \\ \mathcal{N} & \text { Total set of buses } \\ \mathcal{N}_{a} & \text { Set of buses of agent } a \\ \mathcal{N}(n) & \text { Set of neighbors of bus } n \\ {\left[p_{a, n, t}^{\text {min }}, p_{a, n, t}^{\text {max }}\right]} & \text { Minimum and maximum realization } \\ \pi_{t}^{E} & \text { Energy marginal price } \\ \pi_{t}^{I} & \text { Imbalance marginal price } \\ \pi_{t}^{S} & \text { Reservation price of secondary reserve } \\ \pi^{l} & \text { Penalty for a local imbalance } \\ \pi_{i}^{r} & \text { Reservation price of a flexibility service } \\ \pi_{i}^{b} & \text { Activation price of a flexibility service } \\ \pi_{a}^{f} & \text { Retailing price } \\ \pi^{V S P} & \text { Value of shed production } \\ \pi^{V S C} & \text { Value of shed consumption } \\ \mathcal{T} & \text { Set of periods } \\ V_{a, n} & \text { Total energy needs over the horizon } \\ & \end{array}$

Variables.

$\left[b_{a, n}, B_{a, n}\right]$ Safe access bounds to the network

$\mathcal{B}_{a} \quad$ Set of buses where the agent is responsible of its local balance

$\mathcal{E}_{n} \quad$ Set of energy constrained flexibility offers

$\Delta_{a, n, t} \quad$ Total flexibility offered by the FSP

$h_{a, n, t} \quad$ Total requested modulation to the FSP

$I_{a, t} \quad$ Imbalance of an agent

$I_{t} \quad$ Total imbalance of the system

$f_{n_{1}, n_{2}, t} \quad$ Active power flow in line $\left(n_{1}, n_{2}\right)$

$\left[k_{a, n}, K_{a, n}\right] \quad$ Flexible access range of a FSP

$\left[l_{a, n}, L_{a, n}\right] \quad$ Full access range of a FSP

$\left[m_{i, t}, M_{i, t}\right] \quad$ Modulation range of the flexibility service

$p_{a, n, t}^{b} \quad$ Day-ahead baseline of an agent

$P_{n, t}^{b} \quad$ Aggregated day-ahead baseline of the agents

$p_{a, n, t} \quad$ Realization of an agent

$P_{n, t} \quad$ Aggregated realization of the agents

$r_{a, n, t} \quad$ Flexibility needs indicator

$\mathcal{S}_{n} \quad$ Set of single period flexibility offers

$R_{t} \quad$ Flexibility activation needs of the TSO 
$S_{t} \quad$ Flexibility contracted by the TSO

$u_{a, n, t} \quad$ Total requested modulation by the FSU

$v_{a, i} \quad$ Modulation of flexibility service $i \in \mathcal{S}_{n}$

$\left[w_{a, i}, W_{a, i}\right] \quad$ Requested modulation range of flexibility service $i \in \mathcal{E}_{n}$

$x_{a, i, t} \quad$ Modulation of the flexibility service $i \in \mathcal{E}_{n}$

$y_{a, i} \quad$ Binary variable for the reservation of flexibility service $i \in \mathcal{E}_{n}$

$z_{n, t} \quad$ Binary variable equal to 1 if the bus is shed

\subsection{Optimization problems of the Distribution System Operator}

Access agreement. Optimization problem solved to obtain the safe access interval $\left[b_{a, n}, B_{a, n}\right]$ from the access request $\left[g_{a, n}, G_{a, n}\right]$. The objective minimizes the maximum relative access restriction downward and upward, $\delta_{g}$ and $\delta_{G}$, subject to the operational limits of the system, here the line capacities only. Worst case conditions are considered through the auxiliary variables $\underline{f}_{n, m}$ and $\bar{f}_{n, m}$. The first case corresponds to no production and the consumption at its maximal allowed value for each agent and each bus, $b_{a, n}$. The second is equivalent to a case where there is no consumption and all the production are at their maximum bound $B_{a, n}$.

$$
\min \delta_{g}+\delta_{G}
$$

subject to, $\forall n, m \in \mathcal{N}^{2}$,

$$
-C_{n, m} \leq \underline{f}_{n, m}, \bar{f}_{n, m} \leq C_{n, m}
$$

$\forall n \in \mathcal{N}$

$$
\begin{aligned}
\sum_{a \in \mathcal{A}(n)} b_{a, n} & =\sum_{m \in \mathcal{N}(n)} \underline{f}_{n, m} \\
\sum_{a \in \mathcal{A}(n)} B_{a, n} & =\sum_{m \in \mathcal{N}(n)} \bar{f}_{n, m}
\end{aligned}
$$

$\forall n \in \mathcal{N}, a \in \mathcal{A}(n)$

$$
\begin{aligned}
\delta_{g} & \geq\left(g_{a, n}-b_{a, n}\right) / g_{a, n} \\
\delta_{G} & \geq\left(G_{a, n}-B_{a, n}\right) / G_{a, n} \\
g_{a, n} & \leq b_{a, n} \leq 0 \leq B_{a, n} \leq G_{a, n}
\end{aligned}
$$

Announcement of the flexibility needs. The flexibility needs of the DSO in MW, $r_{\mathrm{DSO}, n, t}^{+}$and $r_{\mathrm{DSO}, n, t}^{-}$are obtained by solving

$$
\min \sum_{n \in \mathcal{N}}\left(\alpha_{n, t}^{+} r_{\mathrm{DSO}, n, t}^{+}+\alpha_{n, t}^{-} r_{\mathrm{DSO}, n, t}^{-}\right)
$$

subject to,

$$
-C_{n, m} \leq f_{n, m, t} \leq C_{n, m} \quad \forall n, m \in \mathcal{N}^{2}
$$




$$
\begin{array}{ll}
\sum_{a \in \mathcal{A}} p_{a, n, t}^{b}+r_{\mathrm{DSO}, n, t}^{+}-r_{\mathrm{DSO}, n, t}^{-}=\sum_{m \in \mathcal{N}(n)} f_{n, m, t} & \forall n \in \mathcal{N} \\
r_{\mathrm{DSO}, n, t}^{+}, r_{\mathrm{DSO}, n, t}^{-} \geq 0 & \forall n \in \mathcal{N}
\end{array}
$$

The positive parameters $\alpha_{n}^{+}$and $\alpha_{n}^{-}$characterize the flexibility potential at each bus and are obtained from the DSO knowledge of the system. In the implementation, these parameters are provided by the instance generator.

Flexibility procurement and activation. The DSO can procure a subset of the proposed single period flexibility offers, $\mathcal{S}_{n}$, and energy constrained flexibility offers $\mathcal{E}_{n}$. Let $v_{a, i}$ be the variable for the modulation of the single period flexibility service $i$ and $\left[w_{a, i}, W_{a, i}\right]$ the requested reservation range. Let $x_{a, i, t}^{+}, x_{a, i, t}^{-} \geq 0$ be the variables for the upward and downward activation of the energy constrained flexibility service $i \in \mathcal{E}$. Note that an energy constraint bid cannot be partially contracted which is ensured by the binary variable $y_{a, i}$.

$$
\begin{aligned}
\min & \sum_{i \in \mathcal{S}_{n}}\left(\pi_{i}^{r}\left(W_{D S O, i}-w_{D S O, i}\right)+\pi_{i}^{b} v_{D S O, i}\right) \\
& +\sum_{i \in \mathcal{E}_{n}}\left(\pi_{i}^{r} y_{D S O, i}+\pi_{i}^{b}\left(x_{D S O, i, t}^{+}+x_{D S O, i, t}^{-}\right)\right) \\
+ & \sum_{t \in \mathcal{T}} \sum_{n \in \mathcal{N}} z_{n, t}\left(\pi^{V S P} \max \left\{0, p_{a, n, t}^{b}\right\}+\pi^{V S C} \min \left\{0, p_{a, n, t}^{b}\right\}\right) \\
& +\sum_{t \in \mathcal{T}}\left(\pi_{t}^{I^{+}} I_{D S O, t}^{+}+\pi_{t}^{I^{-}} I_{D S O, t}^{-}\right)
\end{aligned}
$$

subject to

$$
\begin{array}{lr}
-C_{n, m} \leq f_{n, m, t} \leq C_{n, m} & \forall n, m \in \mathcal{N}^{2} \\
\left(1-z_{n, t}\right) \sum_{a \in \mathcal{A}} p_{a, n, t}^{b}+u_{\mathrm{DSO}, n, t}=\sum_{m \in \mathcal{N}(n)} f_{n, m, t} & \forall n \in \mathcal{N}, t \in \mathcal{T} \\
u_{D S O, n, t}=\sum_{i \in \mathcal{S}_{n}: \tau_{i}=t} v_{D S O, i}+\sum_{i \in \mathcal{E}_{n}} x_{D S O, i, t}^{+}-x_{D S O, i, t}^{-} & (7 \mathrm{c}) \\
m_{i} \leq w_{D S O, i} \leq v_{D S O, i} \leq W_{D S O, i} \leq M_{i} & \forall n \in \mathcal{N}, t \in \mathcal{T} \\
m_{i, t} y_{D S O, i} \leq x_{D S O, i, t}^{+}-x_{D S O, i, t}^{-} \leq M_{i, t} y_{D S O, i} & (7 \mathrm{~d}) \\
\sum_{t \in \mathcal{T}}\left(x_{D S O, i, t}^{+}-x_{D S O, i, t}^{-}\right)=0 & \forall n \in \mathcal{N}, i \in \mathcal{S}_{n} \\
(7 \mathrm{e}) \\
(7 \mathrm{f}) \\
\end{array}
$$




$$
\begin{aligned}
& I_{\mathrm{DSO}, t}^{+}-I_{\mathrm{DSO}, t}^{-}=\sum_{n \in \mathcal{N}_{\mathrm{DSO}}} u_{\mathrm{DSO}, n, t} \\
& I_{\mathrm{DSO}, t}^{+}, I_{\mathrm{DSO}, t}^{-} \geq 0
\end{aligned}
$$

The DSO minimizes the cost of flexibility procurement, the estimated cost of shedding production or consumption encoded by the variable $z_{n, t}$ and the imbalance cost caused by the activation of flexibility services. The result of the activation of flexibility services is computed in the variable $u_{D S O, n, t}$ by $(7 \mathrm{~d})$. Constraint $(7 \mathrm{~g})$ ensures that the energy constrained bids are used neutrally in energy. Note that the problem should always be feasible as the DSO could shed every bus of the network i.e. all the production and consumption attached to the bus. This shedding is penalized at a virtual cost $\pi^{V L P}$ for the production and $\pi^{V L C}$ for the consumption.

Real-time operation. A simple protection scheme is represented by the following optimization problem where the only decision variable lefts handle the shedding of buses $z_{n, t}$.

$$
\min \sum_{t \in \mathcal{T}} \sum_{n \in \mathcal{N}} z_{n, t}\left(\pi^{V S P} \max \left\{0, p_{a, n, t}\right\}+\pi^{V S C} \min \left\{0, p_{a, n, t}\right\}\right)
$$

subject to

$$
\begin{array}{rlrl}
-C_{n, m} \leq f_{n, m, t} \leq C_{n, m} & \forall n, m \in \mathcal{N}^{2} \\
\left(1-z_{n, t}\right) \sum_{a \in \mathcal{A}} p_{a, n, t} & =\sum_{m \in \mathcal{N}(n)} f_{n, m, t} & \forall n \in \mathcal{N} .
\end{array}
$$

\subsection{Optimization problems of the producer}

Optimization of the baselines. With the following optimization problem, the producer obtains a baseline for each of its assets considering the following decision stages. We consider that the producer has only one asset by bus. Note that this is not the restrictive assumption as a bus with two assets can be represented as two buses with one asset linked by a line of infinite capacity.

As the flexibility needs of the FSUs cannot be expressed as constraints since the producer may not be willing nor be able to satisfy them, a simple way to translate these needs in the optimization model of the FSP is to define fictive prices which incentivize offering flexibility in the most valuable periods: $\pi_{n, t}^{\Delta^{+}}=$ $\frac{\pi_{n}^{R} R_{n, t}^{+}}{\sum_{t \in \mathcal{T}} R_{n, t}^{+}}$and $\pi_{n, t}^{\Delta^{-}}=\frac{\pi_{n}^{R} R_{n, t}^{-}}{\sum_{t \in \mathcal{T}} R_{n, t}^{-}}$, where $\pi_{n}^{R}$ is a reference flexibility price. This price is a scale factor to quantify the importance of the expected revenue from flexibility with respect to the cost of energy. The flexibility that could be offered upward and downward is denoted $\Delta_{a, n, t}^{+}$and $\Delta_{a, n, t}^{-}$. 


$$
\begin{aligned}
& \max \sum_{t \in \mathcal{T}}\left(\pi_{t}^{E} \sum_{n \in \mathcal{N}_{a}} p_{a, n, t}^{b}-\pi_{t}^{I^{+}} I_{a, t}^{+}-\pi_{t}^{I^{-}} I_{a, t}^{-}\right) \\
& -\sum_{t \in \mathcal{T}} \sum_{n \in \mathcal{N}_{a}} c_{a, n, t} p_{a, n, t}+\sum_{t \in \mathcal{T}}\left(\pi_{n, t}^{\Delta^{+}} \Delta_{a, n, t}^{+}+\pi_{n, t}^{\Delta^{-}} \Delta_{a, n, t}^{-}\right)
\end{aligned}
$$

subject to,

$$
\begin{array}{lr}
k_{a, n} \leq p_{a, n, t}^{b}, p_{a, n, t}, p_{a, n, t}^{+}, p_{a, n, t}^{-} \leq K_{n, t} & \forall n \in \mathcal{N}_{a}, t \in \mathcal{T} \\
I_{a, t}^{+}-I_{a, t}^{-}=\sum_{n \in \mathcal{N}_{a}} p_{a, n, t}-\sum_{n \in \mathcal{N}_{a}} p_{a, n, t}^{b} & \forall t \in \mathcal{T} \\
\Delta_{a, n, t}^{+} \leq p_{a, n, t}^{+}-p_{a, n, t}^{b} & \forall n \in \mathcal{N}_{a}, t \in \mathcal{T} \\
\Delta_{a, n, t}^{-} \leq p_{a, n, t}^{b}-p_{a, n, t}^{-} & \forall n \in \mathcal{N}_{a}, t \in \mathcal{T} \\
p_{a, n, t}^{\min } \leq p_{a, n, t}^{b}, p_{a, n, t}, p_{a, n, t}^{+}, p_{a, n, t}^{-} \leq p_{a, n, t}^{\max } & \forall n \in \mathcal{N}_{a}, t \in \mathcal{T} \\
p_{a, n, t}^{-} \leq L_{a, n} & \forall n \in \mathcal{N}_{a}, t \in \mathcal{T} \\
l_{a, n} \leq p_{a, n, t}^{+} & \forall n \in \mathcal{N}_{a}, t \in \mathcal{T} \\
I_{a, t}^{+}, I_{a, t}^{-} \geq 0 & \forall t \in \mathcal{T}
\end{array}
$$

Optimization of the flexibility offers. The baseline of the producer, $p_{a, n, t}^{b}$ is now fixed. The flexibility available from the producer portfolio is computed using the following optimization problem. If all the baselines of the producer are unchanged with respect to the one computed in the baseline optimization step, the flexibility available is equal to the one predicted in the previous stage.

$$
\begin{aligned}
\max & \sum_{t \in \mathcal{T}}\left(\pi_{n, t}^{\Delta^{+}} \Delta_{a, n, t}^{+}+\pi_{n, t}^{\Delta^{-}} \Delta_{a, n, t}^{-}\right) \\
- & \sum_{t \in \mathcal{T}}\left(\pi_{t}^{I^{+}} I_{a, t}^{+}+\pi_{t}^{I^{-}} I_{a, t}^{-}\right)-\sum_{t \in \mathcal{T}} \sum_{n \in \mathcal{N}_{a}} c_{a, n, t} p_{a, n, t}
\end{aligned}
$$

subject to,

$$
\begin{array}{lr}
p_{a, n, t}^{\min } \leq p_{a, n, t}, p_{a, n, t}^{+}, p_{a, n, t}^{-} \leq p_{a, n, t}^{\max } & \forall n \in \mathcal{N}_{a}, t \in \mathcal{T} \\
I_{a, t}^{+}-I_{a, t}^{-}=\sum_{n \in \mathcal{N}_{a}} p_{a, n, t}-\sum_{n \in \mathcal{N}_{a}} p_{a, n, t}^{b} & \forall t \in \mathcal{T} \\
\Delta_{a, n, t}^{+} \leq p_{a, n, t}^{+}-p_{a, n, t}^{b} & \forall n \in \mathcal{N}_{a}, t \in \mathcal{T} \\
\Delta_{a, n, t}^{-} \leq p_{a, n, t}^{b}-p_{a, n, t}^{-} & \forall n \in \mathcal{N}_{a}, t \in \mathcal{T} \\
k_{a, n} \leq p_{a, n, t}, p_{a, n, t}^{+}, p_{a, n, t}^{-} \leq K_{n, t} & \forall n \in \mathcal{N}_{a}, t \in \mathcal{T} \\
p_{a, n, t}^{-} \leq L_{a, n} & \forall n \in \mathcal{N}_{a}, t \in \mathcal{T} \\
l_{a, n} \leq p_{a, n, t}^{+} & \forall n \in \mathcal{N}_{a}, t \in \mathcal{T}
\end{array}
$$

For each period $t$ and each bus $n$, the producer make one single period flexibility offer upward of $\Delta_{a, n, t}^{+}$at an activation price $c_{a, n, t}$ and one downward of $\Delta_{a, n, t}^{-}$ 
at an activation price $\pi_{t}^{E}-c_{a, n, t}$. The reservation price is a parameter of the simulated instance.

Flexibility services procurement and activation. The producer flexibility procurement optimization problem is similar to the one of the DSO (7) adapted to its specific constraints.

$$
\begin{aligned}
& \min \sum_{i \in \mathcal{S}_{n}}\left(\pi_{i}^{r}\left(W_{a, i}-w_{a, i}\right)+\pi_{i}^{b} v_{a, i}\right)+\sum_{i \in \mathcal{E}_{n}}\left(\pi_{i}^{r} y_{a, i}+\pi_{i}^{b}\left(x_{a, i, t}^{+}+x_{a, i, t}^{-}\right)\right) \\
& +\sum_{t \in \mathcal{T}}\left(\pi_{t}^{I^{+}} I_{a, t}^{+}+\pi_{t}^{I^{-}} I_{a, t}^{-}\right)+\sum_{t \in \mathcal{T}} \sum_{n \in \mathcal{N}_{a}} c_{a, n, t} p_{a, n, t}
\end{aligned}
$$

subject to

$$
\begin{array}{lr}
p_{a, n, t}^{\min } \leq p_{a, n, t} \leq p_{a, n, t}^{\max } & \forall n \in \mathcal{N}_{a}, t \in \mathcal{T} \\
k_{a, n} \leq p_{a, n, t} \leq K_{n, t} & \forall n \in \mathcal{N}_{a}, t \in \mathcal{T} \\
u_{a, n, t}=\sum_{i \in \mathcal{S}_{n}: \tau_{i}=t} v_{a, i}+\sum_{i \in \mathcal{E}_{n}} x_{a, i, t}^{+}-x_{a, i, t}^{-} & \forall n \in \mathcal{N}, t \in \mathcal{T} \\
m_{i} \leq w_{a, i} \leq v_{a, i} \leq W_{a, i} \leq M_{i} & \forall n \in \mathcal{N}, i \in \mathcal{S}_{n} \\
m_{i, t} y_{a, i} \leq x_{a, i, t}^{+}-x_{a, i, t}^{-} \leq M_{i, t} y_{a, i} & \forall n \in \mathcal{N}, i \in \mathcal{E}_{n}, t \in \mathcal{T} \\
\sum_{t \in \mathcal{T}}\left(x_{a, i, t}^{+}-x_{a, i, t}^{-}\right)=0 & \forall n \in \mathcal{N}, i \in \mathcal{E}_{n} \\
I_{a, t}^{+}-I_{a, t}^{-}=\sum_{n \in \mathcal{N}_{a}} p_{a, n, t}-\sum_{n \in \mathcal{N}_{a}}\left(p_{a, n, t}^{b}+u_{a, n, t}\right) & \forall t \in \mathcal{T} \\
I_{a, t}^{+}, I_{a, t}^{-} \geq 0 & \forall t \in \mathcal{T}
\end{array}
$$

Optimization of the realization. The producer chooses the realization considering as parameter flexibility activation requests $h_{a, n, t}$ as a FSP and its flexibility requests as a FSU $u_{a, n, t}$. Note the local imbalance penalty for the buses $n \in \mathcal{B}_{a}$ in which the producer is providing flexibility services.

$\min \sum_{t \in \mathcal{T}}\left(\pi_{t}^{I^{+}} I_{a, t}^{+}+\pi_{t}^{I^{-}} I_{a, t}^{-}\right)+\sum_{t \in \mathcal{T}} \sum_{n \in \mathcal{N}_{a}} c_{a, n, t} p_{a, n, t}+\pi^{l} \sum_{t \in \mathcal{T}} \sum_{n \in \mathcal{B}_{a}}\left(i_{a, n, t}^{+}+i_{a, n, t}^{-}\right)$

subject to

$$
\begin{aligned}
& p_{a, n, t}^{\min } \leq p_{a, n, t} \leq p_{a, n, t}^{\max } \\
& \forall n \in \mathcal{N}_{a}, t \in \mathcal{T}(12 \mathrm{~b}) \\
& k_{a, n} \leq p_{a, n, t} \leq K_{n, t} \\
& \forall n \in \mathcal{N}_{a}, t \in \mathcal{T} \\
& I_{a, t}^{+}-I_{a, t}^{-}=\sum_{n \in \mathcal{N}_{a}} p_{a, n, t}-\sum_{n \in \mathcal{N}_{a}}\left(p_{a, n, t}^{b}+u_{a, n, t}+h_{a, n, t}\right) \\
& \forall t \in \mathcal{T}+E_{a, t} \\
& I_{a, t}^{+}, I_{a, t}^{-} \geq 0 \\
& i_{a, n, t}^{+}-i_{a, n, t}^{-}=p_{a, n, t}-\left(p_{a, n, t}^{b}+u_{a, n, t}+h_{a, n, t}\right) \\
& \forall t \in \mathcal{T}, n \in \mathcal{N}_{a} \\
& i_{a, n, t}^{+}, i_{a, n, t}^{-} \geq 0 \\
& \forall t \in \mathcal{T}, n \in \mathcal{N}_{a}
\end{aligned}
$$$$
\forall t \in \mathcal{T} \quad(12 \mathrm{e})
$$ 


\subsection{Optimization problems of the retailer}

Optimization of the baselines. The retailer baseline optimization problem is similar to the one of the producer (9) adapted to its specific constraints.

$$
\begin{aligned}
& \max \sum_{t \in \mathcal{T}}\left(\pi_{t}^{E} \sum_{n \in \mathcal{N}_{a}} p_{a, n, t}^{b}-\pi_{t}^{I^{+}} I_{a, t}^{+}-\pi_{t}^{I^{-}} I_{a, t}^{-}\right) \\
& -\sum_{t \in \mathcal{T}} \sum_{n \in \mathcal{N}_{a}} c_{a, n, t} p_{a, n, t}+\sum_{t \in \mathcal{T}}\left(\pi_{n, t}^{\Delta^{+}} \Delta_{a, n, t}^{+}+\pi_{n, t}^{\Delta^{-}} \Delta_{a, n, t}^{-}\right)
\end{aligned}
$$

subject to,

$$
\begin{array}{lr}
p_{a, n, t}^{\min } \leq p_{a, n, t}^{b}, p_{a, n, t}, p_{a, n, t}^{+}, p_{a, n, t}^{-} \leq p_{a, n, t}^{\max } & \forall n \in \mathcal{N}_{a}, t \in \mathcal{T} \\
\sum_{t \in \mathcal{T}} p_{a, n, t}=V_{a, n} & \forall n \in \mathcal{N}_{a} \\
I_{a, t}^{+}-I_{a, t}^{-}=\sum_{n \in \mathcal{N}_{a}} p_{a, n, t}-\sum_{n \in \mathcal{N}_{a}} p_{a, n, t}^{b} & \forall t \in \mathcal{T} \\
\Delta_{a, n, t}^{+} \leq p_{a, n, t}^{+}-p_{a, n, t}^{b} & \forall n \in \mathcal{N}_{a}, t \in \mathcal{T} \\
\Delta_{a, n, t}^{-} \leq p_{a, n, t}^{b}-p_{a, n, t}^{-} & \forall n \in \mathcal{N}_{a}, t \in \mathcal{T} \\
k_{a, n} \leq p_{a, n, t}^{b}, p_{a, n, t}, p_{a, n, t}^{+}, p_{a, n, t}^{-} \leq K_{n, t} & \forall n \in \mathcal{N}_{a}, t \in \mathcal{T} \\
p_{a, n, t}^{-} \leq L_{a, n} & \forall n \in \mathcal{N}_{a}, t \in \mathcal{T} \\
l_{a, n} \leq p_{a, n, t}^{+} & \forall n \in \mathcal{N}_{a}, t \in \mathcal{T} \\
I_{a, t}^{+}, I_{a, t}^{-} \geq 0 & \forall t \in \mathcal{T}
\end{array}
$$

Optimization of the flexibility offers. The baseline of the retailer, $p_{a, n, t}^{b}$ is now fixed. The flexibility available from the producer portfolio is computed using the following optimization problem. If all the baselines of the retailer are unchanged with respect to the one computed in the baseline optimization step, the flexibility available is equal to the one predicted in the previous stage.

$$
\begin{aligned}
\max & \sum_{t \in \mathcal{T}}\left(\pi_{n, t}^{\Delta^{+}} \Delta_{a, n, t}^{+}+\pi_{n, t}^{\Delta^{-}} \Delta_{a, n, t}^{-}\right) \\
- & \sum_{t \in \mathcal{T}}\left(\pi_{t}^{I^{+}} I_{a, t}^{+}+\pi_{t}^{I^{-}} I_{a, t}^{-}\right)-\sum_{t \in \mathcal{T}} \sum_{n \in \mathcal{N}_{a}} c_{a, n, t} p_{a, n, t}
\end{aligned}
$$

subject to,

$$
\begin{array}{lr}
k_{a, n} \leq p_{a, n, t}, p_{a, n, t}^{+}, p_{a, n, t}^{-} \leq K_{n, t} & \forall n \in \mathcal{N}_{a}, t \in \mathcal{T} \\
\sum_{t \in \mathcal{T}} p_{a, n, t}=V_{a, n} & \forall n \in \mathcal{N}_{a} \\
I_{a, t}^{+}-I_{a, t}^{-}=\sum_{n \in \mathcal{N}_{a}} p_{a, n, t}-\sum_{n \in \mathcal{N}_{a}} p_{a, n, t}^{b} & \forall t \in \mathcal{T}
\end{array}
$$




$$
\begin{array}{lr}
\Delta_{a, n, t}^{+} \leq p_{a, n, t}^{+}-p_{a, n, t}^{b} & \forall n \in \mathcal{N}_{a}, t \in \mathcal{T} \\
\Delta_{a, n, t}^{-} \leq p_{a, n, t}^{b}-p_{a, n, t}^{-} & \forall n \in \mathcal{N}_{a}, t \in \mathcal{T} \\
p_{a, n, t}^{\min } \leq p_{a, n, t}, p_{a, n, t}^{+}, p_{a, n, t}^{-} \leq p_{a, n, t}^{\max } & \forall n \in \mathcal{N}_{a}, t \in \mathcal{T} \\
p_{a, n, t}^{-} \leq L_{a, n} & \forall n \in \mathcal{N}_{a}, t \in \mathcal{T} \\
l_{a, n} \leq p_{a, n, t}^{+} & \forall n \in \mathcal{N}_{a}, t \in \mathcal{T} \\
I_{a, t}^{+}, I_{a, t}^{-} \geq 0 & \forall t \in \mathcal{T}
\end{array}
$$

For each bus $n$, the retailer makes one energy constrained flexibility offer for the whole time horizon where the maximum and minimum modulation amplitudes are given by $\Delta_{a, n, t}^{+}$and $\Delta_{a, n, t}^{-}$. No activation fee is required by the retailer for its service. The reservation fee is given by

$$
\pi^{R} \cdot \frac{\sum_{t \in \mathcal{T}}\left(\Delta_{a, n, t}^{+}+\Delta_{a, n, t}^{-}\right)}{2}+\sum_{t \in \mathcal{T}} \pi_{t}^{E}\left(p_{a, n, n t}^{b}-p_{a, n, t}^{0}\right)
$$

where the second part is the cost of using the baseline $p_{a, n, t}^{b}$ to increase the available flexibility with respect to a baseline $p_{a, n, t}^{0}$ which is obtained by solving problem (13) without considering flexibility (by setting $\pi_{n, t}^{\Delta^{+}}=\pi_{n, t}^{\Delta^{-}}=0$ ).

Flexibility services procurement and activation. The retailer flexibility procurement optimization problem is similar to the one of the DSO (7) adapted to its specific constraints.

$$
\begin{aligned}
& \min \sum_{i \in \mathcal{S}_{n}}\left(\pi_{i}^{r}\left(W_{a, i}-w_{a, i}\right)+\pi_{i}^{b} v_{a, i}\right)+\sum_{i \in \mathcal{E}_{n}}\left(\pi_{i}^{r} y_{a, i}+\pi_{i}^{b}\left(x_{a, i, t}^{+}+x_{a, i, t}^{-}\right)\right) \\
& +\sum_{t \in \mathcal{T}}\left(\pi_{t}^{I^{+}} I_{a, t}^{+}+\pi_{t}^{I^{-}} I_{a, t}^{-}\right)+\sum_{t \in \mathcal{T}} \sum_{n \in \mathcal{N}_{a}} c_{a, n, t} p_{a, n, t}
\end{aligned}
$$

subject to

$$
\begin{array}{lr}
p_{a, n, t}^{\min } \leq p_{a, n, t} \leq p_{a, n, t}^{\max } & \forall n \in \mathcal{N}_{a}, t \in \mathcal{T} \\
\sum_{t \in \mathcal{T}} p_{a, n, t}=V_{a, n} & \forall n \in \mathcal{N}_{a} \\
k_{a, n} \leq p_{a, n, t} \leq K_{n, t} & \forall n \in \mathcal{N}_{a}, t \in \mathcal{T} \\
u_{a, n, t}=\sum_{i \in \mathcal{S}_{n}: \tau_{i}=t} v_{a, i}+\sum_{i \in \mathcal{E}_{n}} x_{a, i, t}^{+}-x_{a, i, t}^{-} & \forall n \in \mathcal{N}, t \in \mathcal{T} \\
m_{i} \leq w_{a, i} \leq v_{a, i} \leq W_{a, i} \leq M_{i} & \forall n \in \mathcal{N}, i \in \mathcal{S}_{n} \\
m_{i, t} y_{a, i} \leq x_{a, i, t}^{+}-x_{a, i, t}^{-} \leq M_{i, t} y_{a, i} & \forall n \in \mathcal{N}, i \in \mathcal{E}_{n}, t \in \mathcal{T} \\
\sum_{t \in \mathcal{T}}\left(x_{a, i, t}^{+}-x_{a, i, t}^{-}\right)=0 & \forall n \in \mathcal{N}, i \in \mathcal{E}_{n} \\
I_{a, t}^{+}-I_{a, t}^{-}=\sum_{n \in \mathcal{N}_{a}} p_{a, n, t}-\sum_{n \in \mathcal{N}_{a}}\left(p_{a, n, t}^{b}+u_{a, n, t}\right) & \forall t \in \mathcal{T} \\
I_{a, t}^{+}, I_{a, t}^{-} \geq 0 & \forall t \in \mathcal{T}
\end{array}
$$


Optimization of the realization. The retailer chooses the realization considering as parameter flexibility activation requests $h_{a, n, t}$ as a FSP and its flexibility requests as a FSU $u_{a, n, t}$. Note the local imbalance penalty for the buses $n \in \mathcal{B}_{a}$ in which the retailer is providing flexibility services.

$\min \sum_{t \in \mathcal{T}}\left(\pi_{t}^{I^{+}} I_{a, t}^{+}+\pi_{t}^{I^{-}} I_{a, t}^{-}\right)+\sum_{t \in \mathcal{T}} \sum_{n \in \mathcal{N}_{a}} c_{a, n, t} p_{a, n, t}+\pi^{l} \sum_{t \in \mathcal{T}} \sum_{n \in \mathcal{B}_{a}}\left(i_{a, n, t}^{+}+i_{a, n, t}^{-}\right)$

subject to

$$
p_{a, n, t}^{\min } \leq p_{a, n, t} \leq p_{a, n, t}^{\max } \quad \forall n \in \mathcal{N}_{a}, t \in \mathcal{T}
$$

$$
\sum_{t \in \mathcal{T}} p_{a, n, t}=V_{a, n} \quad \forall n \in \mathcal{N}_{a}
$$

$$
\begin{array}{lr}
k_{a, n} \leq p_{a, n, t} \leq K_{n, t} & \forall n \in \mathcal{N}_{a}, t \in \mathcal{T} \\
(17 \mathrm{~d}) \\
I_{a, t}^{+}-I_{a, t}^{-}=\sum_{n \in \mathcal{N}_{a}} p_{a, n, t}-\sum_{n \in \mathcal{N}_{a}}\left(p_{a, n, t}^{b}+u_{a, n, t}+h_{a, n, t}\right)+E_{a, t} & \forall t \in \mathcal{T}
\end{array}
$$

$$
I_{a, t}^{+}, I_{a, t}^{-} \geq 0
$$

$$
i_{a, n, t}^{+}-i_{a, n, t}^{-}=p_{a, n, t}-\left(p_{a, n, t}^{b}+u_{a, n, t}+h_{a, n, t}\right) \quad \forall t \in \mathcal{T}, n \in \mathcal{N}_{a}
$$

$$
i_{a, n, t}^{+}, i_{a, n, t}^{-} \geq 0 \quad \forall t \in \mathcal{T}, n \in \mathcal{N}_{a}
$$

\subsection{Optimization problems of the Transmission System Operator}

Flexibility procurement. The TSO is a pure FSU which flexibility needs $R_{t}^{+}$and $R_{t}^{-}$are taken as data and are not localized. Any FSP may in principle offer up to these amounts to the TSO. The flexibility contracted by the TSO, $S_{t}^{+}$and $S_{t}^{-}$, is obtained by solving (18). As the TSO can procure flexibility outside the distribution system, the amount of flexibility collected in this network weighted by the price difference enters the objective function as a surplus.

$$
\begin{aligned}
\max & \sum_{t \in \mathcal{T}}\left(\pi_{t}^{S^{+}} \min \left\{S_{t}^{+}, R_{t}^{+}\right\}+\pi_{t}^{S^{-}} \max \left\{S_{t}^{-}, R_{t}^{-}\right\}\right) \\
- & \sum_{i \in \mathcal{S}_{n}}\left(\pi_{i}^{r}\left(W_{T S O, i}-w_{T S O, i}\right)+\pi_{i}^{b} v_{a, T S O, i}\right) \\
- & \sum_{i \in \mathcal{E}_{n}}\left(\pi_{i}^{r} y_{T S O, i}+\pi_{i}^{b}\left(x_{T S O, i, t}^{+}+x_{T S O, i, t}^{-}\right)\right)
\end{aligned}
$$


subject to

$$
\begin{array}{lr}
S_{t}^{+}=\sum_{n \in \mathcal{N}} \sum_{i \in \mathcal{S}(n): \tau_{i}=t} W_{T S O, i}+\sum_{n \in \mathcal{N}} \sum_{i \in \mathcal{E}(n)} M_{i, t} y_{T S O, i} & \forall t \in \mathcal{T} \\
S_{t}^{-}=\sum_{n \in \mathcal{N}} \sum_{i \in \mathcal{S}(n): \tau_{i}=t} w_{T S O, i}+\sum_{n \in \mathcal{N}} \sum_{i \in \mathcal{E}(n)} m_{i, t} y_{T S O, i} & (18 \mathrm{~b}) \\
m_{i} \leq w_{T S O, i} \leq v_{T S O, i} \leq W_{T S O, i} \leq M_{i} & \forall t \in \mathcal{T} \\
m_{i, t} y_{T S O, i} \leq x_{T S O, i, t}^{+}-x_{T S O, i, t}^{-} \leq M_{i, t} y_{T S O, i} & (18 \mathrm{c}) \\
\sum_{t \in \mathcal{T}}\left(x_{T S O, i, t}^{+}-x_{T S O, i, t}^{-}\right)=0 & \forall n \in \mathcal{N}, i \in \mathcal{S}_{n} \\
(18 \mathrm{~d}) \\
(18 \mathrm{e}) \\
\end{array}
$$

Flexibility activation. When the TSO decides to activate flexibility services, the TSO solves an imbalance $E_{t}$ which is external to the considered distribution system.

$$
\min \sum_{t \in \mathcal{T}}\left(\pi_{t}^{I^{+}} I_{T S O, t}^{+}+\pi_{t}^{I^{-}} I_{T S O, t}^{-}\right)-\sum_{i \in \mathcal{S}_{n}} \pi_{i}^{b} v_{T S O, i}-\sum_{i \in \mathcal{E}_{n}} \pi_{i}^{b}\left(x_{T S O, i, t}^{+}+x_{T S O, i, t}^{-}\right)
$$

subject to

$$
\begin{aligned}
& E_{T S O, t}+I_{t}^{+}-I_{t}^{-}+\sum_{n \in \mathcal{N}} u_{T S O, n, t}=0 \\
& u_{T S O, n, t}=\sum_{i \in \mathcal{S}_{n}: \tau_{i}=t} v_{T S O, i}+\sum_{i \in \mathcal{E}_{n}} x_{T S O, i, t}^{+}-x_{T S O, i, t}^{-} \\
& I_{T S O, t}^{+}, I_{T S O, t}^{-} \geq 0 \\
& w_{T S O, i} \leq v_{T S O, i} \leq W_{T S O, i} \\
& m_{i, t} y_{T S O, i} \leq x_{T S O, i, t}^{+}-x_{T S O, i, t}^{-} \leq M_{i, t} y_{T S O, i} \\
& \sum_{t \in \mathcal{T}}\left(x_{T S O, i, t}^{+}-x_{T S O, i, t}^{-}\right)=0
\end{aligned}
$$$$
\forall n \in \mathcal{N}, t \in \mathcal{T}
$$

$$
\forall n \in \mathcal{N}, i \in \mathcal{S}_{n}
$$

$\forall n \in \mathcal{N}, i \in \mathcal{E}_{n}$ 


\section{References}

[1] G. Strbac, Demand side management: Benefits and challenges, Energy policy 36 (12) (2008) 4419-4426.

[2] M. H. Albadi, E. El-Saadany, A summary of demand response in electricity markets, Electric Power Systems Research 78 (11) (2008) 1989-1996.

[3] J. Mutale, Benefits of active management of distribution networks with distributed generation, in: Power Systems Conference and Exposition, IEEE/PES, 2006, pp. 601-606.

[4] L. F. Ochoa, C. J. Dent, G. P. Harrison, Distribution network capacity assessment: Variable dg and active networks, Power Systems, IEEE Transactions on 25 (1) (2010) 87-95.

[5] M. Braun, P. Strauss, A review on aggregation approaches of controllable distributed energy units in electrical power systems, International Journal of Distributed Energy Resources 4 (4) (2008) 297-319.

[6] S. Mathieu, D. Ernst, Q. Louveaux, An efficient algorithm for the provision of a day-ahead modulation service by a load aggregator, in: 4th Innovative Smart Grid Technologies, IEEE/PES, 2013.

[7] D. Wang, S. Parkinson, W. Miao, H. Jia, C. Crawford, N. Djilali, Online voltage security assessment considering comfort-constrained demand response control of distributed heat pump systems, Applied Energy 96 (2012) 104-114.

[8] S. Vandael, B. Claessens, M. Hommelberg, T. Holvoet, G. Deconinck, A scalable three-step approach for demand side management of plug-in hybrid vehicles, Smart Grid, IEEE Transactions on 4 (2) (2013) 720-728. doi: 10.1109/TSG. 2012.2213847.

[9] S. Widergren, J. Sun, L. Tesfatsion, Market design test environments, in: Power Engineering Society General Meeting, 2006, IEEE, 2006, pp. 6-pp.

[10] S. D. McArthur, E. M. Davidson, V. M. Catterson, A. L. Dimeas, N. D. Hatziargyriou, F. Ponci, T. Funabashi, Multi-agent systems for power engineering applications - part i: Concepts, approaches, and technical challenges, Power Systems, IEEE Transactions on 22 (4) (2007) 1743-1752.

[11] S. D. McArthur, E. M. Davidson, V. M. Catterson, A. L. Dimeas, N. D. Hatziargyriou, F. Ponci, T. Funabashi, Multi-agent systems for power engineering applications - part ii: technologies, standards, and tools for building multi-agent systems, Power Systems, IEEE Transactions on 22 (4) (2007) 1753-1759.

[12] Z. Zhou, W. K. V. Chan, J. H. Chow, Agent-based simulation of electricity markets: a survey of tools, Artificial Intelligence Review 28 (4) (2007) 305342. 
[13] M. Ventosa, A. Ballo, A. Ramos, M. Rivier, Electricity market modeling trends, Energy policy 33 (7) (2005) 897-913.

[14] A. Weber, J.-M. Glachant, V. Rious, M. Saguan, J. B. Gil, E. R. Puente, L. Kitzing, P. E. Morthorst, S. T. Schröder, et al., Optimate-a modeling breakthrough for market design analysis to test massive intermittent generation integration in markets, in: 9th International Conference on the European Energy Market, 2012.

[15] S. Mathieu, Q. Louveaux, D. Ernst, B. Cornélusse, A quantitative analysis of the effect of flexible loads on reserve markets, in: 18th Power Systems Computation Conference, IEEE, 2014.

[16] P. Capros, L. Mantzos, N. Tasios, A. De Vita, N. Kouvaritakis, EU Energy Trends to 2030: Update 2009, Publications Office of the European Union, 2010.

[17] R. Belhomme, M. Sebastian, A. Diop, M. Entem, F. Bouffard, G. Valtorta, A. De Simone, R. Cerero, C. Yuen, S. Karkkainen, W. Fritz, Address technical and commercial conceptual architectures (10).

[18] Q. Gemine, E. Karangelos, D. Ernst, B. Cornélusse, Active network management: planning under uncertainty for exploiting load modulation, in: Bulk Power System Dynamics and Control-IX Optimization, Security and Control of the Emerging Power Grid (IREP), 2013 IREP Symposium, IEEE, 2013, pp. 1-9.

[19] iPower, Development of a DSO-market on flexibility services (4 2013).

[20] A. I. Ramos Gutierrez, E. Rivero Puente, D. Six, evolvdso - development of methodologies and tools for new and evolving dso roles for efficient dres integration in distribution networks (2014).

[21] B. Dupont, P. Vingerhoets, P. Tant, K. Vanthournout, W. Cardinaels, T. De Rybel, E. Peeters, R. Belmans, LINEAR breakthrough project: Large-scale implementation of smart grid technologies in distribution grids, in: Innovative Smart Grid Technologies (ISGT Europe), 2012 3rd IEEE PES International Conference and Exhibition on, 2012, pp. 1-8. doi: 10.1109/ISGTEurope.2012.6465708.

[22] Adine project.

URL http://www.hermia.fi/in_english/services/ coordination-of-programmes-and-p/adine/results/

[23] S. Koch, M. Zima, G. Andersson, Active coordination of thermal household appliances for load management purposes, in: IFAC Symposium on Power Plants and Power Systems Control, Tampere, Finland, Citeseer, 2009. 
[24] A. Michiorri, R. Girard, G. Kariniotakis, C. Lebosse, S. Albou, A local energy management system for solar integration and improved security of supply: The Nice Grid project, 2012.

[25] M. Maenhoudt, G. Deconinck, Agent-based modelling as a tool for testing electric power market designs, in: International Conference on the European Energy Market, 2010.

[26] S. Gill, I. Kockar, G. W. Ault, Dynamic optimal power flow for active distribution networks, Power Systems, IEEE Transactions on 29 (1) (2014) $121-131$.

[27] J. P. Lopes, N. Hatziargyriou, J. Mutale, P. Djapic, N. Jenkins, Integrating distributed generation into electric power systems: A review of drivers, challenges and opportunities, Electric Power Systems Research 77 (9) (2007) 1189-1203.

[28] T. Achterberg, Scip: solving constraint integer programs, Mathematical Programming Computation 1 (1) (2009) 1-41.

[29] SEDG, Uk generic distribution system (ukgds) project (2010).

URL www.sedg.ac.uk

[30] ELIA (2014). [link].

URL www.elia.be

[31] Belpex (2014). [link].

URL www. belpex.be 